\title{
Electronic (Absorption) Spectra of 3d Transition Metal Complexes
}

\author{
S. Lakshmi Reddy, Tamio Endo and G. Siva Reddy
}

Additional information is available at the end of the chapter

http://dx.doi.org/10.5772/50128

\section{Introduction}

\subsection{Types of spectra}

Spectra are broadly classified into two groups (i) emission spectra and (ii) absorption spectra

i. Emission spectra Emission spectra are of three kinds (a) continuous spectra,(b) band spectra and (c) line spectra.

Continuous spectra: Solids like iron or carbon emit continuous spectra when they are heated until they glow. Continuous spectrum is due to the thermal excitation of the molecules of the substance.

Band spectra: The band spectrum consists of a number of bands of different colours separated by dark regions. The bands are sharply defined at one edge called the head of the band and shade off gradually at the other edge. Band spectrum is emitted by substances in the molecular state when the thermal excitement of the substance is not quite sufficient to break the molecules into continuous atoms.

Line spectra: A line spectrum consists of bright lines in different regions of the visible spectrum against a dark background. All the lines do not have the same intensity. The number of lines, their nature and arrangement depends on the nature of the substance excited. Line spectra are emitted by vapours of elements. No two elements do ever produce similar line spectra.

ii. Absorption spectra: When a substance is placed between a light source and a spectrometer, the substance absorbs certain part of the spectrum. This spectrum is called the absorption spectrum of the substance. 
Electronic absorption spectrum is of two types. $d-d$ spectrum and charge transfer spectrum. $\mathrm{d}-\mathrm{d}$ spectrum deals with the electronic transitions within the d-orbitals. In the charge transfer spectrum, electronic transitions occur from metal to ligand or vice-versa.

\section{Electronic spectra of transitions metal complexes}

Electronic absorption spectroscopy requires consideration of the following principles:

a. Franck-Condon Principle: Electronic transitions occur in a very short time (about $10^{-15}$ sec.) and hence the atoms in a molecule do not have time to change position appreciably during electronic transition .So the molecule will find itself with the same molecular configuration and hence the vibrational kinetic energy in the exited state remains the same as it had in the ground state at the moment of absorption.

b. Electronic transitions between vibrational states: Frequently, transitions occur from the ground vibrational level of the ground electronic state to many different vibrational levels of particular excited electronic states. Such transitions may give rise to vibrational fine structure in the main peak of the electronic transition. Since all the molecules are present in the ground vibrational level, nearly all transitions that give rise to a peak in the absorption spectrum will arise from the ground electronic state. If the different excited vibrational levels are represented as $v_{1}, v_{2}$, etc., and the ground state as $v_{0}$, the fine structure in the main peak of the spectrum is assigned to $v_{0} \rightarrow v_{0}, v_{0} \rightarrow v_{1}, v_{0} \rightarrow v_{2}$ etc., vibrational states. The $v_{0} \rightarrow v_{0}$ transition is the lowest energy (longest wave length) transition.

c. Symmetry requirement: This requirement is to be satisfied for the transitions discussed above.

Electronic transitions occur between split ' $d$ ' levels of the central atom giving rise to so called d-d or ligand field spectra. The spectral region where these occur spans the near infrared, visible and U.V. region.

$\begin{array}{llll}\text { Ultraviolet (UV) } & \text { Visible (Vis) } & \text { Near infrared (NIR) } & \\ 50,000-26300 & 26300-12800 & 12800-5000 & \mathrm{~cm}^{-1} \\ 200-380 & 380-780 & 780-2000 & \mathrm{~nm}\end{array}$

\section{Russel-Saunders or L-S coupling scheme}

An orbiting electronic charge produces magnetic field perpendicular to the plane of the orbit. Hence the orbital angular momentum and spin angular momentum have corresponding magnetic vectors. As a result, both of these momenta couple magnetically to give rise to total orbital angular momentum. There are two schemes of coupling: RusselSaunders or L-S coupling and j-j coupling.

a. The individual spin angular momenta of the electrons, $\mathrm{Si}$, each of which has a value of \pm $1 / 2$, combine to give a resultant spin angular momentum (individual spin angular momentum is represented by a lower case symbol whereas the total resultant value is given by a upper case symbol). 


$$
\sum s_{i}=S
$$

Two spins of each $\pm 1 / 2$ could give a resultant value of $S=1$ or $S=0$; similarly a resultant of three electrons is $1 \frac{1 / 2}{2}$ or $1 / 2$. The resultant is expressed in units of $h / 2 \pi$. The spin multiplicity is given by $(2 \mathrm{~S}+1)$. Hence, If $\mathrm{n}$ is the number of unpaired electrons, spin multiplicity is given by $n+1$.

b. The individual orbital angular momenta of electrons, $l_{i}$, each of which may be $0,1,2,3$, $4 \ldots$. in units of $\mathrm{h} / 2 \pi$ for $\mathrm{s}, \mathrm{p}, \mathrm{d}, \mathrm{f}, \mathrm{g}, \ldots . .$. orbitals respectively, combine to give a resultant orbital angular momentum, $\mathrm{L}$ in units of $\mathrm{h} / 2 \pi . \quad \sum \mathrm{l}_{\mathrm{i}}=\mathrm{L}$

The resultant $\mathrm{L}$ may be once again $0,1,2,3,4 \ldots$ which are referred to as $S, P, D, F$ G, . respectively in units of $h / 2 \pi$. The orbital multiplicity is given by $(2 L+1)$.

$\begin{array}{llllll}0 & 1 & 2 & 3 & 4 & 5 \\ \text { S } & \text { P } & \text { D } & \text { F } & \text { G } & \text { H }\end{array}$

c. Now the resultant S and L couple to give a total angular momentum, J. Hence, it is not surprising that $\mathrm{J}$ is also quantized in units of $\mathrm{h} / 2 \pi$. The possible values of $\mathrm{J}$ quantum number are given as

$$
\mathrm{J}=(\mathrm{L}+\mathrm{S}),(\mathrm{L}+\mathrm{S}-1),(\mathrm{L}+\mathrm{S}-2),(\mathrm{L}+\mathrm{S}-3), \ldots . .|\mathrm{L}-\mathrm{S}|,
$$

The symbol | I indicates that the absolute value $(\mathrm{L}-\mathrm{S})$ is employed, i.e., no regard is paid to \pm sign. Thus for $L=2$ and $S=1$, the possible $J$ states are 3,2 and 1 in units of $h / 2 \pi$.

The individual spin angular momentum, si and the individual orbital angular momentum, $\mathrm{l}_{\mathrm{i}}$, couple to give total individual angular momentum, $\mathrm{j}$ i. This scheme of coupling is known as spin-orbit coupling or $\mathrm{j}-\mathrm{j}$ coupling.

\section{Term symbols}

\subsection{Spectroscopic terms for free ion ground states}

The rules governing the term symbol for the ground state according to L-S coupling scheme are given below:

a. The spin multiplicity is maximized i.e., the electrons occupy degenerate orbitals so as to retain parallel spins as long as possible (Hund's rule).

b. The orbital angular momentum is also maximized i.e., the orbitals are filled with highest positive $\mathrm{m}$ values first.

c. If the sub-shell is less than half-filled, $\mathrm{J}=\mathrm{L}-\mathrm{S}$ and if the sub-shell is more than half filled, $\mathrm{J}=\mathrm{L}+\mathrm{S}$.

The term symbol is given by ${ }^{25+1} \mathrm{LJ}$. The left-hand superscript of the term is the spin multiplicity, given by $2 \mathrm{~S}+1$ and the right- hand subscript is given by J. It should be noted that $S$ is used to represent two things- (a) total spin angular momentum and (b) and total angular momentum when $\mathrm{L}=0$. The above rules are illustrated with examples. 
For $\mathrm{d}^{4}$ configuration:

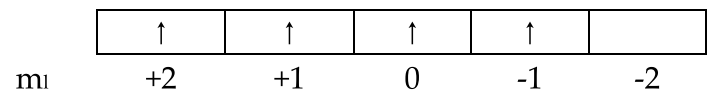

Hence, $\mathrm{L}=3-1=2$ i.e., $\mathrm{D} ; \mathrm{S}=2 ; 2 \mathrm{~S}+1=5$; and $\mathrm{J}=\mathrm{L}-\mathrm{S}=0$; Term symbol $={ }^{5} \mathrm{D}_{0}$

For $\mathrm{d}^{9}$ configuration:

$\mathrm{ml} \quad$\begin{tabular}{|c|c|c|c|c|}
\hline$\downarrow \uparrow$ & $\downarrow \uparrow$ & $\downarrow \uparrow$ & $\downarrow \uparrow$ & $\uparrow$ \\
\hline+2 & +1 & 0 & -1 & -2
\end{tabular}

Hence, $\mathrm{L}=+2+1+0-1=2$ i.e., $\mathrm{D} ; \mathrm{S}=1 / 2 ; 2 \mathrm{~S}+1=2$; and $\mathrm{J}=\mathrm{L}+\mathrm{S}=3 / 2$; Term symbol $={ }^{2} \mathrm{D}_{5 / 2}$

Spin multiplicity indicates the number of orientations in the external field. If the spin multiplicity is three, there will be three orientations in the magnetic field.- parallel, perpendicular and opposed. There are similar orientations in the angular momentum in an external field.

The spectroscopic term symbols for $\mathrm{d}^{\mathrm{n}}$ configurations are given in the Table- 1 . The terms are read as follows: The left-hand superscript of the term symbol is read as singlet, doublet, triplet, quartet, quintet, sextet, septet, octet, etc., for spin multiplicity values of 1, 2, 3, 4, 5, 6, 7 , 8, etc., respectively. ${ }^{1} \mathrm{~S}_{0}$ (singlet $\mathrm{S}$ nought); ${ }^{2} \mathrm{~S}_{1 / 2}$ (doublet $\mathrm{S}$ one-half); ${ }^{3} \mathrm{P}_{2}$ (triplet $\mathrm{P}$ two ); ${ }^{5} \mathrm{I}_{8}$ (quintet I eight). It is seen from the Table- 1 that $\mathrm{d}^{\mathrm{n}}$ and $\mathrm{d}^{10-\mathrm{n}}$ have same term symbols, if we ignore J values. Here $\mathrm{n}$ stands for the number of electrons in $\mathrm{d}^{\mathrm{n}}$ configuration.

\begin{tabular}{|c|c|c|c|}
\hline $\mathrm{d}^{\mathrm{n}}$ & Term & $\mathrm{d}^{\mathrm{n}}$ & Term \\
\hline $\mathrm{d}^{0}$ & ${ }^{1} \mathrm{~S}_{0}$ & $\mathrm{~d}^{10}$ & ${ }^{1} \mathrm{~S}_{0}$ \\
$\mathrm{~d}^{1}$ & ${ }^{2} \mathrm{D}_{3 / 2}$ & $\mathrm{~d}^{9}$ & ${ }^{2} \mathrm{D}_{5 / 2}$ \\
$\mathrm{~d}^{2}$ & ${ }^{3} \mathrm{~F}_{2}$ & $\mathrm{~d}^{8}$ & ${ }^{3} \mathrm{~F}_{4}$ \\
$\mathrm{~d}^{4}$ & ${ }^{5} \mathrm{D}_{0}$ & $\mathrm{~d}^{6}$ & ${ }^{5} \mathrm{D}_{4}$ \\
$\mathrm{~d}^{5}$ & ${ }^{6} \mathrm{~S}_{5 / 2}$ & & \\
\hline
\end{tabular}

Table 1. Term symbols

It is also found that empty sub -shell configurations such as $\mathrm{p}^{0}, \mathrm{~d}^{0}, \mathrm{f}^{0}$, etc., and full filled subshell configurations such as $\mathrm{p}^{6}, \mathrm{~d}^{10}, \mathrm{f}^{14}$, etc., have always the term symbol ${ }^{1} \mathrm{~S}_{0}$ since the resultant spin and angular momenta are equal to zero. All the inert gases have term symbols for their ground state ${ }^{1} S_{0}$.Similarly all alkali metals reduce to one electron problems since closed shell core contributes nothing to $\mathrm{L}, \mathrm{S}$ and $\mathrm{J}$; their ground state term symbol is given by ${ }^{2} S_{1 / 2}$. Hence $d$ electrons are only of importance in deciding term symbols of transition metals.

\section{Total degeneracy}

We have seen that the degeneracy with regard to spin is its multiplicity which is given by $(2 \mathrm{~S}+1)$. The total spin multiplicity is denoted by $\mathrm{M}_{\mathrm{s}}$ running from $\mathrm{S}$ to $-\mathrm{S}$. Similarly orbital 
degeneracy, $\mathrm{M}_{\mathrm{L}}$, is given by $(2 \mathrm{~L}+1)$ running from $\mathrm{L}$ to $-\mathrm{L}$. For example, $\mathrm{L}=2$ for $\mathrm{D}$ state and so the orbital degeneracy is $(2 \times 2+1)=5$ fold. Similarly, for F state, the orbital degeneracy is seven fold. Since there are $(2 \mathrm{~L}+1)$ values of $\mathrm{ML}_{\mathrm{L}}$ and $(2 \mathrm{~S}+1)$ values of $\mathrm{M}_{\mathrm{s}}$ in each term, the total degeneracy of the term is given by: $2(\mathrm{~L}+1)(2 \mathrm{~S}+1)$.

Each value of ML occurs (2S+1) times and each value of $M_{s}$ occurs (2L+1) times in the term. For ${ }^{3} \mathrm{~F}$ state, the total degeneracy is $3 \times 7=21$ fold and for the terms ${ }^{3} \mathrm{P},{ }^{1} \mathrm{G},{ }^{1} \mathrm{D},{ }^{1} \mathrm{~S}$, the total degeneracy is $9,9,5,1$ fold respectively. Each fold of degeneracy represents one microstate.

\section{Number of microstates}

The electrons may be filled in orbitals by different arrangements since the orbitals have different $\mathrm{m}$ values and electrons may also occupy singly or get paired. Each different type of electronic arrangement gives rise to a microstate. Thus each electronic configuration will have a fixed number of microstates. The numbers of microstates for $\mathrm{p}^{2}$ configuration are given in Table-2 (for both excited and ground states).

\begin{tabular}{|l|r|r|r|r|r|r|r|r|r|r|r|r|r|r|r|}
\hline $\begin{array}{l}\mathrm{m} \iota \\
-1\end{array}$ & & $\uparrow$ & $\uparrow$ & & $\downarrow$ & $\downarrow$ & & $\downarrow$ & $\downarrow$ & & & $\uparrow$ & & & $\uparrow \downarrow$ \\
\hline 0 & $\uparrow$ & & $\uparrow$ & $\downarrow$ & & $\downarrow$ & $\downarrow$ & & $\uparrow$ & $\uparrow$ & $\uparrow$ & $\downarrow$ & & $\uparrow \downarrow$ & \\
\hline+1 & $\uparrow$ & $\uparrow$ & & $\downarrow$ & $\downarrow$ & & $\uparrow$ & $\uparrow$ & & $\downarrow$ & $\downarrow$ & & $\uparrow \downarrow$ & & \\
\hline $\mathrm{mL}$ & +1 & 0 & -1 & +1 & 0 & -1 & +1 & 0 & -1 & +1 & +1 & -1 & +2 & 0 & \\
\hline
\end{tabular}

Table 2. Number of microstates for $\mathrm{p}^{2}$ configuration

Each vertical column is one micro state. Thus for $\mathrm{p}^{2}$ configuration, there are 15 microstates. In the above diagram, the arrangement of singlet states of paired configurations given in $\mathrm{A}$ (see below) is not different from that given in B and hence only one arrangement for each $\mathrm{m}$ value.
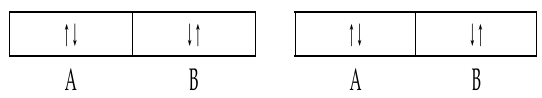

The number of microstates possible for any electronic configuration may be calculated from the formula,

Number of microstates $=n ! / r !(n-r)$ !

Where $\mathrm{n}$ is the twice the number of orbitals, $\mathrm{r}$ is the number of electrons and ! is the factorial.

For $\mathrm{p}^{2}$ configuration, $\mathrm{n}=3 \times 2=6 ; \mathrm{r}=2 ; \mathrm{n}-\mathrm{r}=4$

$6 !=6 \times 5 \times 4 \times 3 \times 2 \times 1=720 ; 2 !=2 \times 1=2 ; 4 !=4 \times 3 \times 2 \times 1=24$

Substituting in the formula, the number of microstates is 15 . 
Similarly for a $\mathrm{d}^{2}$ configuration, the number of microstates is given by 10 ! / 2 ! $(10-2)$ !

$$
\frac{10 \times 9 \times 8 \times 7 \times 6 \times 5 \times 4 \times 3 \times 2 \times 1}{2 \times 1(8 \times 7 \times 6 \times 5 \times 4 \times 3 \times 2 \times 1)}=45
$$

Thus a $\mathrm{d}^{2}$ configuration will have 45 microstates. Microstates of different $\mathrm{d}^{\mathrm{n}}$ configuration are given in Table-3.

\begin{tabular}{|l|c|c|c|c|c|c|}
\hline $\mathrm{d}^{\mathrm{n}}$ configuration & $\mathrm{d}^{1}, \mathrm{~d}^{9}$ & $\mathrm{~d}^{2}, \mathrm{~d}^{8}$ & $\mathrm{~d}^{3}, \mathrm{~d}^{7}$ & $\mathrm{~d}^{4}, \mathrm{~d}^{6}$ & $\mathrm{~d}^{5}$ & $\mathrm{~d}^{10}$ \\
\hline No.of microstates & 10 & 45 & 120 & 210 & 252 & 1 \\
\hline
\end{tabular}

Table 3. Microstates of different $\mathrm{d}^{\mathrm{n}}$ configuration

\section{Multiple term symbols of excited states}

The terms arising from $\mathrm{d}^{\mathrm{n}}$ configuration for $3 \mathrm{~d}$ metal ions are given Table- 4 .

\begin{tabular}{|c|c|c|}
\hline Configuration & Ion & Term symbol \\
\hline $\mathrm{d}^{1}$ & $\mathrm{Ti}^{3+}, \mathrm{V}^{4+}$ & \\
\hline$d^{9}$ & $\mathrm{Cu}^{2+}$ & ${ }^{2} \mathrm{D}$ \\
\hline$d^{2}$ & $\mathrm{Ti}^{2+}, \mathrm{V}^{3+}, \mathrm{Cr}^{4+}$ & \\
\hline$d^{8}$ & $\mathrm{Ni}^{2+}$ & ${ }^{3} \mathrm{~F},{ }^{3} \mathrm{P},{ }^{1} \mathrm{G},{ }^{1} \mathrm{D},{ }^{1} \mathrm{~S}$ \\
\hline$d^{3}$ & $\mathrm{Cr}^{3+}, \mathrm{V}^{2+}, \mathrm{Mn}^{4+}$ & \\
\hline$d^{7}$ & $\mathrm{Ni}^{3+}, \mathrm{Co}^{2+}$ & ${ }^{4} \mathrm{~F},{ }^{4} \mathrm{P},{ }^{2}(\mathrm{H}, \mathrm{G}, \mathrm{F}, \mathrm{D}, \mathrm{D}, \mathrm{P})$ \\
\hline $\mathrm{d}^{4}$ & $\mathrm{Cr}^{2+}, \mathrm{Mn}^{3+}$ & \\
\hline$d^{6}$ & $\mathrm{Fe}^{2+}, \mathrm{Co}^{3+}$ & ${ }^{5} \mathrm{D},{ }^{3}(\mathrm{H}, \mathrm{G}, \mathrm{F}, \mathrm{F}, \mathrm{D}, \mathrm{P}, \mathrm{P}),{ }^{1}(\mathrm{I}, \mathrm{G}, \mathrm{G}, \mathrm{F}, \mathrm{D}, \mathrm{D}, \mathrm{S}, \mathrm{S})$ \\
\hline$d^{5}$ & $\mathrm{Mn}^{2+}, \mathrm{Fe}^{3+}$ & \\
\hline$d^{10}$ & $\mathrm{Zn}^{2+}$ & $\begin{array}{l}{ }^{6} \mathrm{~S},{ }^{4}(\mathrm{G}, \mathrm{F}, \mathrm{D}, \mathrm{P}),{ }^{2}(\mathrm{I}, \mathrm{H}, \mathrm{G}, \mathrm{G}, \mathrm{F}, \mathrm{F}),{ }^{2}(\mathrm{D}, \mathrm{D}, \mathrm{D}, \mathrm{P}, \mathrm{S}) \\
{ }^{6} \mathrm{~S}\end{array}$ \\
\hline
\end{tabular}

Table 4. Terms arising from $\mathrm{d}^{\mathrm{n}}$ configuration for $3 \mathrm{~d}$ ions ( $\mathrm{n}=1$ to10) 


\section{Selection rules}

\subsection{La Porte selection rule}

This rule says that transitions between the orbitals of the same sub shell are forbidden. In other words, the for total orbital angular momentum is $\Delta \mathrm{L}= \pm 1$. This is La Porte allowed transitions. Thus transition such as ${ }^{1} \mathrm{~S} \rightarrow{ }^{1} \mathrm{P}$ and ${ }^{2} \mathrm{D} \rightarrow{ }^{2} \mathrm{P}$ are allowed but transition such as ${ }^{3} \mathrm{D} \rightarrow 3 \mathrm{~S}$ is forbidden since $\Delta \mathrm{L}=-2$.That is, transition should involve a change of one unit of angular momentum. Hence transitions from gerade to ungerade ( $g$ to $u$ ) or vice versa are allowed, i.e., $u \rightarrow g$ or $g \rightarrow u$ but not $u \rightarrow u$ or $g \rightarrow g$. In the case of $\mathrm{p}$ sub shell, both ground and excited states are odd and in the case of $d$ sub shell both ground and excited states are even. As a rule transition should be from even to odd or vice versa.

The same rule is also stated in the form of a statement instead of an equation:

Electronic transitions within the same $p$ or $d$ sub-shell are forbidden, if the molecule has centre of symmetry.

\subsection{Spin selection rule}

The selection Rule for Spin Angular Momentum is

$$
\Delta S=0
$$

Thus transitions such as ${ }^{2} \mathrm{~S} \rightarrow{ }^{2} \mathrm{P}$ and ${ }^{3} \mathrm{D} \rightarrow{ }^{3} \mathrm{P}$ are allowed, but transition such as ${ }^{1} \mathrm{~S} \rightarrow 3 \mathrm{P}$ is forbidden. The same rule is also stated in the form of a statement,

Electronic Transitions between the different states of spin multiplicity are forbidden.

The selection Rule for total angular momentum, J, is

$$
\Delta \mathrm{J}=0 \text { or } \pm 1
$$

The transitions such as ${ }^{2} \mathrm{P}_{1 / 2} \rightarrow{ }^{2} \mathrm{D}_{3 / 2}$ and ${ }^{2} \mathrm{P}_{3 / 2} \rightarrow{ }^{2} \mathrm{D}_{3 / 2}$ are allowed, but transition such as ${ }^{2} \mathrm{P}_{1 / 2} \rightarrow{ }^{2} \mathrm{D}_{5 / 2}$ is forbidden since $\Delta \mathrm{J}=2$.

There is no selection rule governing the change in the value of $n$, the principal quantum number. Thus in hydrogen, transitions such as $1 \mathrm{~s} \rightarrow 2 \mathrm{p}, 1 \mathrm{~s} \rightarrow 3 \mathrm{p}, 1 \mathrm{~s} \rightarrow 4 \mathrm{p}$ are allowed.

Usually, electronic absorption is indicated by reverse arrow, $\leftarrow$, and emission is indicated by the forward arrow, $\rightarrow$, though this rule is not strictly obeyed.

\subsection{Mechanism of breakdown of selection rules}

\subsubsection{Spin-orbit coupling}

For electronic transition to take place, $\Delta S=0$ and $\Delta \mathrm{L}= \pm 1$ in the absence of spin-orbit coupling. However, spin and orbital motions are coupled. Even, if they are coupled very weakly, a little of each spin state mixes with the other in the ground and excited states by an amount dependent 
upon the energy difference in the orbital states and magnitude of spin -orbit coupling constant. Therefore electronic transitions occur between different states of spin multiplicity and also between states in which $\Delta \mathrm{L}$ is not equal to \pm 1 . For example, if the ground state were $99 \%$ singlet and $1 \%$ triplet (due to spin- orbit coupling) and the excited state were $1 \%$ singlet and 99 $\%$ triplet, then the intensity would derive from the triplet -triplet and singlet-singlet interactions. Spin-orbit coupling provides small energy differences between degenerate state.

This coupling is of two types. The single electron spin orbit coupling parameter $\zeta$, gives the strength of the interaction between the spin and orbital angular momenta of a single electron for a particular configuration. The other parameter, $\lambda$, is the property of the term. For high spin complexes,

$$
\lambda= \pm \xi / 2 S
$$

Here positive sign holds for shells less than half field and negative sign holds for more than half filled shells. $S$ is the same as the one given for the free ion. The $\lambda$ values in crystals are close to their free ion values. $\Lambda$ decreases in crystal with decreasing Racah parameters $\mathrm{B}$ and C. For high spin $\mathrm{d}^{5}$ configuration, there is no spin orbit coupling because ${ }^{6} \mathrm{~S}$ state is unaffected by the ligand fields. The $\lambda$ and $\zeta$ values for $3 d$ series are given in Table- 5 .

\begin{tabular}{|l|c|c|c|c|c|c|c|}
\hline Ion & Ti(II) & V(II) & Cr(II) & Mn(II) & Fe(II) & Co(II) & Ni(II) \\
\hline$\Xi\left(\mathrm{cm}^{-1}\right)$ & 121 & 167 & 230 & 347 & 410 & 533 & 649 \\
\hline$\lambda\left(\mathrm{cm}^{-1}\right)$ & 60 & 56 & 57 & 0 & -102 & -177 & -325 \\
\hline
\end{tabular}

Table 5. $\lambda$ and $\zeta$ values for $3 d$ series

\subsubsection{La Porte selection rule}

Physically $3 \mathrm{~d}$ (even) and $4 \mathrm{p}$ (odd) wave functions may be mixed, if centre of inversion (i) is removed. There are two processes by which $\mathrm{i}$ is removed.

a. The central metal ion is placed in a distorted field (tetrahedral field, Tetragonal distortions, etc.,) The most important case of distorted or asymmetric field is the case of a tetrahedral complex. Tetrahedron has no inversion centre and so d-p mixing takes place. So electronic transitions in tetrahedral complexes are much more intense, often by a factor 100, than in a analogous octahedral complexes. Trans isomer of $\left[\mathrm{Co}(\mathrm{en})_{2} \mathrm{Cl}_{2}\right]^{+}$ in aqueous solution is three to four times less intense than the cis isomer because the former is centro-symmetric. Other types of distortion include Jahn-Teller distortions.

b. Odd vibrations of the surrounding ligands create the distorted field for a time that is long enough compared to the time necessary for the electronic transition to occur (Franck Condon Principle).Certain vibrations will remove the centre of symmetry. Mathematically this implies coupling of vibrational and electronic wave functions. Breaking down of La Porte rule by vibrionic coupling has been termed as "Intensity Stealing". If the forbidden excited term lies energetically nearby a fully allowed transition, it would produce a very intense band. Intensity Stealing by this mechanism decreases in magnitude with increasing energy separation between the excited term and the allowed level. 


\section{Splitting of energy states}

The symbols A(or $\mathbf{a}$ ) and $\mathbf{B}$ (or $\mathbf{b}$ ) with any suffixes indicate wave functions which are singly degenerate. Similarly $\mathbf{E}$ (or $\mathbf{e}$ ) indicates double degeneracy and $\mathbf{T}$ (or $\mathbf{t}$ ) indicates triple degeneracy. Lower case symbols, $\mathbf{a}$ 1g, $\mathbf{a} \mathbf{2}$, $\mathbf{e}_{\mathbf{g}}$, etc., are used to indicate electron wave functions(orbitals) and upper case symbols are used to describe electronic energy levels. Thus ${ }^{2} \mathbf{T}_{2 \mathrm{~g}}$ means an energy level which is triply degenerate with respect to orbital state and also doubly degenerate with respect to its spin state. Upper case symbols are also used without any spin multiplicity term and they then refer to symmetry (ex., A1g symmetry). The subscripts $\mathbf{g}$ and $\mathbf{u}$ indicate gerade (even) and ungerade (odd).

$\mathbf{d}$ orbitals split into two sets $-\mathbf{t}_{2 g}$ orbitals and $\mathbf{e}_{\mathrm{g}}$ orbitals under the influence crystal field. These have $\mathbf{T}_{\mathbf{2 g}}$ and $\mathbf{E}_{\mathrm{g}}$ symmetry respectively. Similarly $\mathbf{f}$ orbitals split into three sets - $\mathbf{a}_{\mathbf{2}} \mathbf{u}$

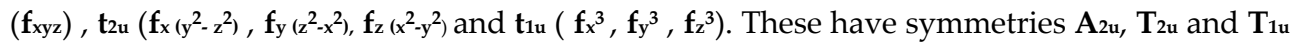
respectively.

Splitting of $\mathbf{D}$ state parallels the splitting of $\mathbf{d}$ orbitals and splitting of $\mathbf{F}$ state splits parallels splitting of $\mathbf{f}$ orbitals. For example, $\mathbf{F}$ state splits into either $\mathbf{T}_{1} \mathbf{u}, \mathbf{T}_{2} \mathbf{u}$ and $\mathbf{A}_{2 \mathbf{z}}$ or $\mathbf{T}_{1 \mathrm{~g}}, \mathbf{T}_{2 \mathrm{~g}}$ and $\mathbf{A}_{2 \mathrm{~g}}$ sub-sets. Which of these is correct is determined by $\mathbf{g}$ or $\mathbf{u}$ nature of the configuration from which $\mathbf{F}$ state is derived. Since $\mathbf{f}$ orbitals are $\mathbf{u}$ in character ${ }^{2} \mathbf{F}$ state corresponding to $\mathbf{f}^{1}$ configuration splits into ${ }^{2} \mathbf{T}_{1 u},{ }^{2} \mathbf{T}_{2 u}$, and ${ }^{2} \mathbf{A}_{2 u}$ components; similarly ${ }^{3} \mathbf{F}$ state derived from $\mathbf{d}^{2}$ configuration splits into ${ }^{3} \mathbf{T}_{2 \mathrm{~g}},{ }^{3} \mathbf{T}_{1 \mathrm{~g}}$ and ${ }^{3} \mathbf{A}_{2 \mathrm{~g}}$ components because $\mathbf{d}$ orbitals are $\mathbf{g}$ in character.

\subsection{Splitting of energy states corresponding to $\mathrm{d}^{\mathrm{n}}$ terms}

These are given in Table-6.

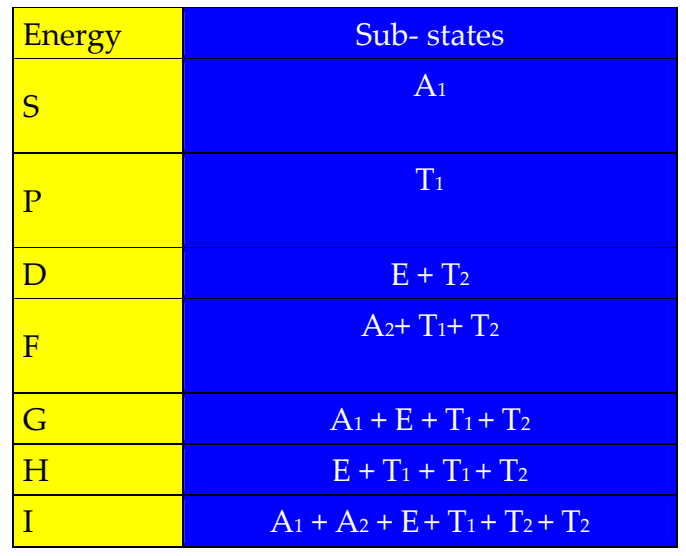

Table 6. Splitting of energy states corresponding to $\mathrm{d}^{\mathrm{n}}$ terms

The $\mathrm{d}$ - $\mathrm{d}$ spectra is concerned with $\mathrm{d}^{\mathrm{n}}$ configuration and hence the crystal field sub-states are given for all the $\mathrm{d}^{\mathrm{n}}$ configuration in Table -7 . 


\begin{tabular}{|c|c|c|c|c|}
\hline Configuration & $\begin{array}{c}\text { Free ion } \\
\text { ground state }\end{array}$ & $\begin{array}{l}\text { Crystal field } \\
\text { substates }\end{array}$ & $\begin{array}{c}\text { Important } \\
\text { excited } \\
\text { states }\end{array}$ & $\begin{array}{c}\text { Crystal field } \\
\text { state }\end{array}$ \\
\hline$d^{1}, d^{9}$ & ${ }^{2} \mathrm{D}$ & ${ }^{2} \mathrm{~T}_{2 \mathrm{~g}},{ }^{2} \mathrm{Eg}$ & & \\
\hline $\mathrm{d}^{2}, \mathrm{~d}^{8}$ & ${ }^{3} \mathrm{~F}$ & ${ }^{3} \mathrm{~T}_{1 \mathrm{~g}},{ }^{3} \mathrm{~T}_{2 \mathrm{~g},}{ }^{3} \mathrm{~A}_{2 \mathrm{~g}}$ & ${ }^{3} \mathrm{P}$ & ${ }^{3} \mathrm{~T}_{1 \mathrm{~g}}$ \\
\hline$d^{3}, d^{7}$ & ${ }^{4} \mathrm{~F}$ & ${ }^{4} \mathrm{~T}_{1 \mathrm{~g}},{ }^{4} \mathrm{~T}_{2 \mathrm{~g},}{ }^{4} \mathrm{~A}_{2 \mathrm{~g}}$ & ${ }^{4} \mathrm{p}$ & ${ }^{4} \mathrm{~T}_{1 \mathrm{~g}}$ \\
\hline$d^{4}, d^{6}$ & ${ }^{5} \mathrm{D}$ & ${ }^{5} \mathrm{~T}_{2 \mathrm{~g}},{ }^{5} \mathrm{Eg}$ & & \\
\hline$d^{5}$ & ${ }^{6} \mathrm{~S}$ & ${ }^{6} \mathrm{~A}_{1 \mathrm{~g}}$ & & \\
\hline
\end{tabular}

Table 7. Crystal field components of the ground and some excited states of $d^{n}(n=1$ to 9 ) configuration

\section{Energy level diagram}

Energy Level Diagrams are described by two independent schemes - Orgel Diagrams which are applicable to weak field complexes and Tanabe -Sugano (or simply T-S) Diagrams which are applicable to both weak field and strong field complexes.

\section{Inter-electronic repulsion parameters}

The inter-electronic repulsions within a configuration are linear combinations of Coulombic and exchange integrals above the ground term. They are expressed by either of the two ways: Condon - Shortley parameters, $\mathrm{F}_{0}, \mathrm{~F}_{2}$ and $\mathrm{F}_{4}$ and Racah parameters, A, B and C. The magnitude of these parameters varies with the nature of metal ion.

\subsection{Racah parameters}

The Racah parameters are A, B and C. The Racah parameter A corresponds to the partial shift of all terms of a given electronic configuration. Hence in the optical transition considerations, it is not taken into account. The parameter, B measures the inter electronic repulsion among the electrons in the d-orbitals. The decrease in the value of the interelectronic repulsion parameter, B leads to formation of partially covalent bonding. The ratio between the crystal $\mathrm{B}^{1}$ parameter and the free ion $\mathrm{B}$ parameter is known as nephelauxetic rato and it is denoted by $\beta$. The value of $\beta$ is a measure of covalency. The smaller the value, the greater is the covalency between the metal ion and the ligands. The $\mathrm{B}$ and $\mathrm{C}$ values are a measure of spatial arrangement of the orbitals of the ligand and the metal ion.

Racah redefined the empirical Condon -Shortley parameters so that the separation between states having the maximum multiplicity (for example, difference between is a function of ${ }^{3} \mathrm{~F}$ and ${ }^{3} \mathrm{P}$ or ${ }^{4} \mathrm{~F}$ and ${ }^{4} \mathrm{P}$ is a function of a single parameter, $\mathrm{B}$. However, separations between terms of different multiplicity involve both B and C 


\section{Tanabe -Sugano diagrams}

Exact solutions for the excited sate energy levels in terms of Dq, B and C are obtained from Tanabe-Sugano matrices. However, these are very large $(10 \times 10)$ matrices and hand calculations are not feasible. For this reason Tanabe-Sugano have drawn energy level diagrams known as T-S diagrams or energy level diagrams. The T-S diagrams are valid only if the value of $\mathrm{B}, \mathrm{C}$ and $\mathrm{Dq}$ ae lower for a complex than for the free ion value.

Quantitative interpretation of electronic absorption spectra is possible by using Tanabe Sugano diagrams or simply T-S diagrams. These diagrams are widely employed to correlate and interpret spectra for ions of all types, from $\mathrm{d}^{2}$ to $\mathrm{d}^{8}$. Orgel diagrams are useful only qualitatively for high spin complexes whereas T-S diagrams are useful both for high spin and low spin complexes. The x-axis in T-S diagrams represent the ground state term. Further, in T-S diagrams, the axes are divided by $\mathrm{B}$, the interelectronic repulsion parameter or Racah Parameter. The $\mathrm{x}$-axis represents the crystal field strength in terms of Dq/ B or $\Delta / \mathrm{B}$ and the $\mathrm{Y}$-axis represents the energy in terms of $\mathrm{E} / \mathrm{B}$.

The energies of the various electronic states are given in the T-S diagrams on the vertical axis and the ligand field strength increases from left to right on the horizontal axis. The symbols in the diagram omit the subscript, g, with the understanding that all states are gerade states. Also, in T.S. diagrams, the zero of energy for any particular $\mathrm{d}^{\mathrm{n}}$ ion is taken to be the energy of the ground state. Regardless of the ligand field strength, then, the horizontal axis represents the energy of the ground state because the vertical axis is in units of $\mathrm{E} / \mathrm{B}$ and $\mathrm{x}$-axis is also in units of $\Delta / \mathrm{B}$. Thus, the unit of energy in T-S diagram is $\mathrm{B}, \mathrm{Racah}$ Parameter.

The values of $B$ are different for different ions of the same $\mathrm{d}^{\mathrm{n}}$ (or different $\mathrm{d}^{\mathrm{n}}$ configuration) which is shown on the top of each diagram. One T-S diagram is used for all members of an isoelectronic group. Also some assumption is made about the relative value of C/B.

\section{Electron spin resonance}

Electron Spin Resonance (ESR) is a branch of spectroscopy in which radiation of microwave frequency is absorbed by molecules possessing electrons with unpaired spins. It is known by different names such as Electron Paramagnetic Resonance (EPR), Electron Spin Resonance (ESR) and Electron Magnetic Resonance (EMR). This method is an essential tool for the analysis of the structure of molecular systems or ions containing unpaired electrons, which have spin-degenerate ground states in the absence of magnetic field. In the study of solid state materials, EPR method is employed to understand the symmetry of surroundings of the paramagnetic ion and the nature of its bonding to the nearest neighbouring ligands.

When a paramagnetic substance is placed in a steady magnetic field $(\mathrm{H})$, the unpaired electron in the outer shell tends to align with the field. So the two fold spin degeneracy is 
removed. Thus the two energy levels, $E_{1 / 2}$ and $\mathrm{E}_{-1 / 2}$ are separated by $\mathrm{g} \beta \mathrm{H}$, where $\mathrm{g}$ is spectroscopic splitting factor and is called gyro magnetic ratio and $\beta$ is the Bohr magneton. Since there is a finite probability for a transition between these two energy levels, a change in the energy state can be stimulated by an external radio frequency. When microwave frequency $(v)$ is applied perpendicular to the direction of the field, resonance absorption will occur between the two split spin levels. The resonance condition is given by, $h v=g \beta H$, where $\mathrm{h}$ is Planck's constant.

The resonance condition can be satisfied by varying $v$ or $H$. However, EPR studies are carried out at a constant frequency $(v)$, by varying magnetic field $(\mathrm{H})$. For a free electron, the $\mathrm{g}$ value is 2.0023 . Since $\mathrm{h}$ and $\beta$ are constants, one can calculate the $\mathrm{g}$ factor. This factor determines the divergence of the Zeeman levels of the unpaired electron in a magnetic field and is characteristic of the spin system.

In the crystal systems, the electron spins couple with the orbital motions and the $g$ value is a measure of the spin and orbital contributions to the total magnetic moment of the unpaired electron and any deviation of magnetic moment from the free spin value is due to the spinorbit interaction. It is known that the crystal field removes only the orbital degeneracy of the ground terms of the central metal ion either partially or completely. The strong electrical fields of the surrounding ligands results in "Stark Splitting" of the energy levels of the paramagnetic ion. The nature and amount of splitting strongly depends on the symmetry of the crystalline electric field. The Stark splitting of the free ion levels in the crystal field determines the magnetic behaviour of the paramagnetic ion in a crystal. Whenever there is a contribution from the unquenched orbital angular momentum, the measured $g$ values are isotropic as a result of the asymmetric crystal field since the contribution from the orbital motion is anisotropic. To decide the ultimate ground state of a paramagnetic ion in the crystal, the two important theorems, Kramers and Jahn-Teller, are useful. Using group theory, one can know the nature of the splitting of the free ion levels in the crystal fields of various symmetries.

Jahn-Teller theorem states that any nonlinear molecule in an electronically degenerate ground state is unstable and tends to distort in order to remove this degeneracy. The direction of distortion which results in greatest stabilization can often be deduced from EPR and other spectroscopic data.

Kramers' theorem deals with restrictions to the amount of spin degeneracy which can be removed by a purely electrostatic field. If the system contains an odd number of electrons, such an electrostatic field cannot reduce the degeneracy of any level below two. Each pair forms what is known as a Kramers' doublet, which can be separated only by a magnetic field. For example, Fe(III) and Mn(II) belonging to $\mathrm{d}^{5}$ configuration, exhibit three Kramers' doublets labeled as $| \pm 5 / 2>,| \pm 3 / 2>$ and $\mid \pm 1 / 2>$.

If the central metal ion also possesses a non-zero nuclear spin, I, then hyperfine splitting occurs as a result of the interaction between the nuclear magnetic moment and the electronic magnetic moment. The measurement of $g$ value and hyperfine splitting factor provides 
information about the electronic states of the unpaired electrons and also about the nature of the bonding between the paramagnetic ion and its surrounded ligands. If the ligands also contain non-zero nuclear spin, then the electron spin interacts with the magnetic moment of the ligands. Then one could expect super hyperfine EPR spectrum.

The $g$ value also depends on the orientation of the molecules having the unpaired electron with respect to the applied magnetic field. In the case of perfect cubic symmetry, the $g$ value does not depend on the orientation of the crystal. But in the case of low symmetry crystal fields, $g$ varies with orientation. Therefore we get three values $g_{x x}, g_{y y}$, and $g_{z z}$ corresponding to $a, b$ and $c$ directions of the crystal. In the case of tetragonal site $g_{x x}=g_{y y}$ which is referred to as $g_{\perp}$ and corresponds to the external magnetic field perpendicular to the $\mathrm{Z}$-axis. When it is parallel, the value is denoted as $\mathrm{g}_{\|}$. Hence one can deduce the symmetry of a complex by EPR spectrum i.e., cubic, tetragonal, trigonal or orthorhombic. Anyhow, it is not possible to distinguish between orthorhombic and other lower symmetries by EPR.

\subsection{EPR signals of first group transition metal ions}

Transition metal ions of $3 \mathrm{~d}$ group exhibit different patterns of EPR signals depending on their electron spin and the crystalline environment. For example, $3 \mathrm{~d}^{1}$ ions, $\mathrm{VO}^{2+}$ and $\mathrm{Ti}^{3+}$ have $s=1 / 2$ and hence are expected to exhibit a single line whose $g$ value is slightly below 2.0. In the case of most abundant ${ }^{51} \mathrm{~V}, \mathrm{~s}=1 / 2$ and $\mathrm{I}=7 / 2$, an eight line pattern with hyperfine structure of almost equal intensity can be expected as shown in Fig-1. In the case of most abundant $\mathrm{Ti},(\mathrm{s}=1 / 2$ and $\mathrm{I}=0)$, no hyperfine structure exists. However, the presence of less abundant isotopes $\left({ }^{47} \mathrm{Ti}\right.$ with $\mathrm{I}=5 / 2$ and ${ }^{49} \mathrm{Ti}$ with $\mathrm{I}=7 / 2$ ) give rise to weak hyperfine structure with six and eight components respectively. This weak structure is also shown in Fig-1.

$\mathrm{Cr}(\mathrm{III})$, a d $\mathrm{d}^{3}$ ion, with $\mathrm{s}=3 / 2$ exhibits three fine line structure. The most abundant ${ }^{52} \mathrm{Cr}$ has $\mathrm{I}=0$ and does not exhibit hyperfine structure. However, ${ }^{53} \mathrm{Cr}$ with $\mathrm{I}=3 / 2$ gives rise to hyperfine structure with four components. This structure will be weak because of the low abundance of ${ }^{53} \mathrm{Cr}$. Thus each one of the three fine structure lines of ${ }^{53} \mathrm{Cr}$ is split into four weak hyperfine lines. Of these, two are overlapped by the intense central line due to the most abundant ${ }^{52} \mathrm{Cr}$ and the other two lines are seen in the form of weak satellites.

$\mathrm{Mn}(\mathrm{II})$ and $\mathrm{Fe}(\mathrm{III})$ with $\mathrm{d}^{5}$ configuration have $\mathrm{s}=5 / 2$ and exhibit five lines which correspond

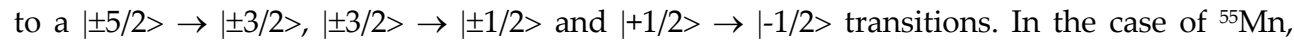
which has $I=5 / 2$, each of the five transitions will give rise to a six line hyperfine structure. But in powders, usually one observes the six-hyperfine lines corresponding to $|+1 / 2>\rightarrow|-$ $1 / 2>$ transition only. The remaining four transition sets will be broadened due to the high anisotropy. $\mathrm{Fe}^{3+}$ yields no hyperfine structure as seen in Fig -1 .

$\mathrm{Co}^{2+}$, a d $\mathrm{d}^{7}$ configuration, with $\mathrm{s}$ value of $3 / 2$ exhibits three fine structure lines. In the case of ${ }^{59} \mathrm{Co}(\mathrm{I}=7 / 2)$, eight line hyperfine pattern can be observed as shown in the Fig-1. 


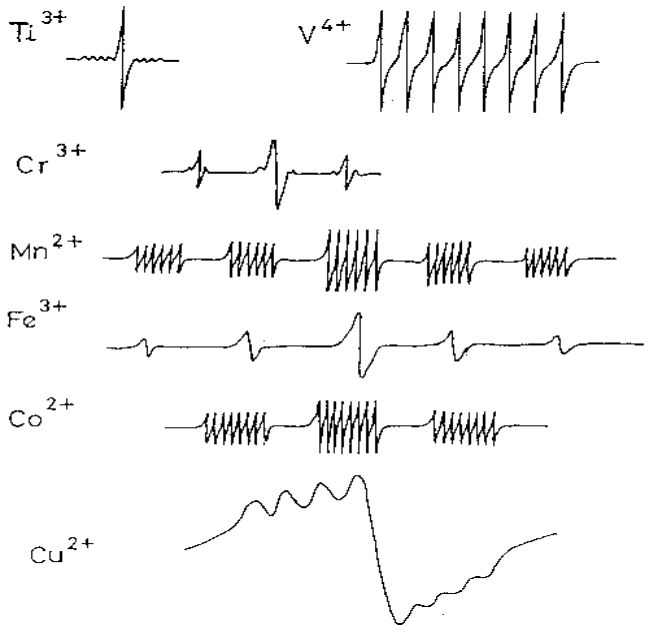

Figure 1. EPR signal of $3 d$ ions

\section{Survey of experimental results}

\subsection{Titanium}

Titanium is the ninth most abundant element in the Earth's crust $(0.6 \%)$. There are 13 known isotopes of titanium. Among them five are natural isotopes with atomic masses 46 to 50 and the others are artificial isotopes. The synthetic isotopes are all radioactive. Titanium alloys are used in spacecraft, jewelry, clocks, armored vehicles, and in the construction of buildings. The compounds of titanium are used in the preparation of paints, rubber, plastics, paper, smoke screens $\left(\mathrm{TiCl}_{4}\right.$ is used), sunscreens. The main sources of Ti are ilmenite and rutile.

Titanium exhibits +1 to +4 ionic states. Among them $\mathrm{Ti}^{4+}$ has $\mathrm{d}^{0}$ configuration and hence has no unpaired electron in its outermost orbit. Thus $\mathrm{Ti}^{4+}$ exhibits diamagnetism. Hence no d-d transitions are possible. The ionic radius of $\mathrm{Ti}^{3+}$ is the same as that of $\mathrm{Fe}(\mathrm{II})$ (0.76 A.U). Ti(I) and $\mathrm{Ti}(\mathrm{III})$ have unpaired electrons in their outermost orbits and exhibit para magnetism

\subsection{Electronic spectra of titanium compounds}

The electronic configuration of $\mathrm{Ti}^{3}$ is [Ar] $3 \mathrm{~d}^{1} 4 \mathrm{~s}^{2}$. It has five fold degeneracy and its ground state term symbol is ${ }^{2} \mathrm{D}$. In an octahedral crystal field, the five fold degeneracy is split into ${ }^{2} \mathrm{~T}_{2 \mathrm{~g}}$ and ${ }^{2} \mathrm{Eg}$ states. Thus only one single electron transition, ${ }^{2} \mathrm{~T}_{2 \mathrm{~g}} \rightarrow{ }^{2} \mathrm{Eg}$, is expected in an octahedral crystal field. The separation between these energies is $10 \mathrm{Dq}$, which is crystal field energy. Normally, the ground ${ }^{2} \mathrm{~T}_{2 \mathrm{~g}}$ state is split due to Jahn-Teller effect and hence lowering of symmetry is expected for $\mathrm{Ti}(\mathrm{III})$ ion. This state splits into ${ }^{2} \mathrm{~B}_{2 \mathrm{~g}}$ and ${ }^{2} \mathrm{Eg}$ states in tetragonal symmetry and the excited term ${ }^{2} \mathrm{Eg}_{\mathrm{g}}$ also splits into ${ }^{2} \mathrm{~B}_{1 \mathrm{~g}}$ and ${ }^{2} \mathrm{~A} 1 \mathrm{~g}$ levels. Thus, three bands are expected for tetragonal $\left(C_{4 v}\right)$ symmetry. Energy level diagram in tetragonal environment is shown in Fig -2. 


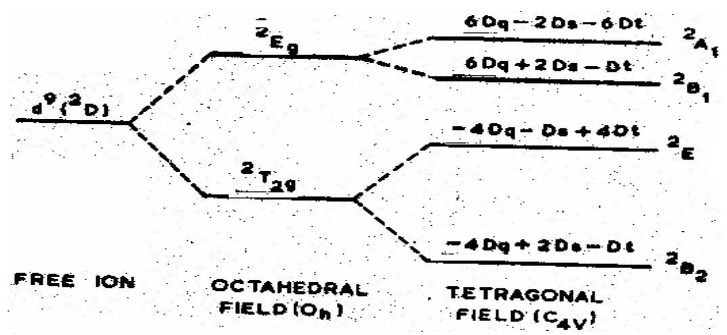

Figure 2. Energy level diagram of $\mathrm{Ti}^{3+}$ in octahedral and tetragonal fields

The transitions in the tetragonal field are described by the following equations.

$$
\begin{gathered}
{ }^{2} B_{2 g} \rightarrow{ }^{2} E_{g}:[-4 D q-D s+4 D t-(-4 D q+2 D s-D t)]=-3 D s+5 D t \\
B_{2 g} \rightarrow{ }^{2} B_{1 g}:[6 D q+2 D s-D t-(-4 D q+2 D s-D t)]=10 D q \\
{ }^{2} B_{2 g} \rightarrow{ }^{2} A_{1 g}:[6 D q-2 D s-6 D t-(-4 D q+2 D s-D t)]=10 D q-4 D s+5 D t
\end{gathered}
$$

In the above formulae, Dq is octahedral crystal field and Ds and Dt are tetragonal field parameters. The same sign of $\mathrm{Dq}$ and $\mathrm{Dt}$ indicates an axial elongation and opposite sign indicates an axial compression

\subsubsection{EPR spectra of titanium compounds}

When any $\mathrm{Ti}(\mathrm{III})$ compound in the form of powder is placed in a magnetic field, it gives a resonance signal. The single d-electron of $\mathrm{Ti}^{3+}$ has spin, $\mathrm{s}=1 / 2$. The abundance of isotopes is reported as ${ }^{46} \mathrm{Ti} \approx 87 \%,{ }^{48} \mathrm{Ti} \approx 7.7 \%$ and ${ }^{50} \mathrm{Ti} \approx 5.5 \%$ and have nuclear spin $\mathrm{I}=0,5 / 2$ and $7 / 2$ respectively. Electron spin and nuclear spin interactions give rise to (2I+1) hyperfine lines $(0,6$ and 8$)$ and appear as satellite. Since ${ }^{46} \mathrm{Ti}$ abundance is more, the EPR signal contains only one resonance line which is similar to the one shown in Fig-3. The g value for this resonance is slightly less than 2.0 .

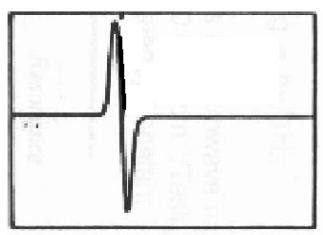

Figure 3. RT powered EPR spectrum of Ti(III).

\subsubsection{Relation between EPR and optical absorption spectra}

EPR studies for $\mathrm{Ti}^{3+}$ can be correlated with optical data to obtain the orbital reduction parameter. 


$$
\alpha=K \frac{\lambda_{(\text {Covalency) }}}{\lambda_{\text {(Ionic) }}}=\frac{\left(g_{e}-g_{11}\right) \Delta E}{n \lambda_{\text {(ionic) }}}
$$

where $\mathrm{n}$ is 8 for $\mathrm{C}_{4 \mathrm{v}}, \Delta E$ is the energy of appropriate transition, $\lambda$ is the spin-orbit coupling constant for $\mathrm{Ti}^{3+}$, i.e., $154 \mathrm{~cm}^{-1}$ and $\mathrm{k}$ is the orbital reduction parameter.

\subsubsection{Typical examples}

EPR and optical absorption spectral data of selected samples are discussed as examples. The data chosen from the literature are typical for each sample and hence should be considered as representative only. For more complete information on specific example, the original references are to be consulted. X-band spectra and optical absorption spectra of the powdered samples are recorded at room temperature (RT).

\subsubsection{Optical absorption studies}

$\mathrm{Ti}(\mathrm{III})$ ion in solids is characterized by three broad bands around 7000, 12000 and $18000 \mathrm{~cm}^{-1}$. These are due to the transitions from ${ }^{2} \mathrm{~B}_{2 g} \rightarrow{ }^{2} \mathrm{E}_{g},{ }^{2} \mathrm{~B}_{2 g} \rightarrow{ }^{2} \mathrm{~B}_{1 g}$, and ${ }^{2} \mathrm{~B}_{2 g} \rightarrow{ }^{2} \mathrm{~A}_{1 g}$ respectively. Three bands of titanite at 7140, 13700 and $16130 \mathrm{~cm}^{-1}$ and of anatase at 6945,12050 and $18180 \mathrm{~cm}^{-1}$ are attributed to the above transitions. The optical absorption spectrum of lamprophyllite is also similar. The optical absorption spectrum of benitoite sample displays three bands at 8260 , 10525 and $15880 \mathrm{~cm}^{-1}$. From the observed band positions, the crystal field parameter in octahedral field, Dq and tetragonal field parameters, Ds and Dt, are given in Table-8.

\begin{tabular}{|l|l|l|l|}
\hline Sample & $\mathrm{Dq} \mathrm{cm}^{-1}$ & $\mathrm{Ds} \mathrm{cm}^{-1}$ & $\mathrm{Dt} \mathrm{cm}^{-1}$ \\
\hline Titanite & 1370 & -1367 & 608 \\
\hline Anatase & 1205 & -1867 & 268 \\
\hline Lamprophyllite & 877 & -1426 & 1525 \\
\hline Benitoite & 1050 & -1945 & 485 \\
\hline
\end{tabular}

Table 8. Crystal field parameters of $\mathrm{Ti}(\mathrm{III})$

The magnitude of Dt indicates the strength of the tetragonal distortion. This is more in lamprophyllite when compared to the other samples.

i. X-band EPR spectra of the powdered sample of titanite shows a broad resonance line in the centre $(335.9 \mathrm{mT})$. The measured $\mathrm{g}$ value is 1.957 . Another resonance line is noticed at $341.4 \mathrm{mT}$ with $\mathrm{g}=1.926$. The central eight line transition is superimposed on the spectrum and the components are attributed to $\mathrm{VO}$ (II) impurity. The $\mathrm{g}$ value of $\mathrm{Ti}^{3+}$ is 1.957 and other $g$ value is due to $\mathrm{VO}(\mathrm{II})$. The $\mathrm{g}$ value of 1.95 indicates that $\mathrm{Ti}^{3+}$ is in tetragonally distorted octahedral site.

ii. The EPR spectrum of anatase shows a large number of resonances centered around $\mathrm{g}$ value of 2 which is attributed to $\mathrm{Ti}^{3+}$. The additional structures between $\mathrm{g}$ values of 2 and 4 are attributed to $\mathrm{Fe}(\mathrm{III})$ impurity in the compound. Both the ions are in tetragonally distorted environment. 
iii. X band EPR of polycrystalline lamprophyllite sample indicates a broad resonance line with line width $56.6 \mathrm{mT}$ and a $\mathrm{g}$ value of 2.0. This is due to the presence of Ti(III) in the compound. The broad line is due to the dipolar-dipolar interaction of $\mathrm{Ti}(\mathrm{III})$ ions. Even at liquid nitrogen temperature, only the line intensity increases indicating that Curie law is obeyed.

Using EPR and optical absorption spectral results of titanite, the covalency parameter is calculated using equation (4), $\alpha=\frac{\left(g_{e}-g_{11}\right) \Delta E}{n \lambda_{\text {(ionic) }}}$. The $\alpha$ value obtained is 0.51 , which indicates higher covalent character between ligand and metal ion.

\section{Vanadium}

Vanadium abundance in earth's crust is 120 parts per million by weight. Vanadium's ground state electron configuration is [Ar] $3 \mathrm{~d}^{3} 4 \mathrm{~s}^{2}$. Vanadium exhibits four common oxidation states $+5,+4,+3$, and +2 each of which can be distinguished by its color. Vanadium $(\mathrm{V})$ compounds are yellow in color whereas +4 compounds are blue, +3 compounds are green and +2 compounds are violet in colour. Vanadium is used in making specialty steels like rust resistant and high speed tools. The element occurs naturally in about 65 different minerals and in fossil fuel deposits. Vanadium is used by some life forms as an active center of enzymes. Vanadium oxides exhibit intriguing electrochemical, photochemical, catalytical, spectroscopic and optical properties. Vanadium has 18 isotopes with mass numbers varying from 43 to 60 . Of these, ${ }^{51} \mathrm{~V}$, natural isotope is stable:

\subsection{Electronic spectra of vanadium compounds}

Vanadium in its tetravalent state invariably exists as oxo-cation, $\mathrm{VO}^{2+}$ (vanadyl). The $\mathrm{VO}^{2+}$ ion has a single $d$ electron which gives rise to the free ion term ${ }^{2} \mathrm{D}$. In a crystal field of octahedral symmetry, this electron occupies the $t_{2 g}$ orbital and gives rise to ground state term ${ }^{2} \mathrm{~T}_{2 \mathrm{~g}}$. When the electron absorbs energy, it is excited to the eg orbital and accordingly in octahedral geometry only one band corresponding to the transition, ${ }^{2} \mathrm{~T}_{2 g} \rightarrow{ }^{2} \mathrm{Eg}$, is expected. Because of the non-symmetrical alignment of the $\mathrm{V}=\mathrm{O}$ bond along the axis, the site symmetry, in general, is lowered to tetragonal $\left(\mathrm{C}_{4 \mathrm{~V}}\right)$ or rhombic $\left(\mathrm{C}_{2 \mathrm{~V}}\right)$ symmetry. In $\mathrm{C}_{4 \mathrm{~V}}$ site symmetry, ${ }^{2} \mathrm{~T}_{2 \mathrm{~g}}$ splits into ${ }^{2} \mathrm{~B}_{2 g}$ and ${ }^{2} \mathrm{Eg}$, whereas ${ }^{2} \mathrm{Eg}_{\mathrm{g}}$ splits into ${ }^{2} \mathrm{~B}_{1 \mathrm{~g}},{ }^{2} \mathrm{~A}_{1 \mathrm{~g}}$. Hence three bands are expected in $\mathrm{C}_{4 \mathrm{v}}$ symmetry in the range of $11000-14000,14500-19000$ and $20000-31250 \mathrm{~cm}^{-1}$. The degeneracy of ${ }^{2} \mathrm{Eg}$ is also removed in $\mathrm{C}_{2 \mathrm{~V}}$ symmetry resulting four bands. Energy level diagram of $\mathrm{VO}^{2+}$ in octahedral $\mathrm{C}_{4 \mathrm{v}}$ and $\mathrm{C}_{2 \mathrm{v}}$ symmetries are shown in Fig- 4 . In the tetragonal $\mathrm{C}_{4 \mathrm{v}}$ symmetry transitions are described by the following equations.

$$
\begin{gathered}
{ }^{2} B_{2 g} \rightarrow{ }^{2} E_{g}:[-4 D q-D s+4 D t-(-4 D q+2 D s-D t)]=-3 D s+5 D t \\
B_{2 g} \rightarrow{ }^{2} B_{1 g}:[6 D q+2 D s-D t-(-4 D q+2 D s-D t)]=10 D q
\end{gathered}
$$




$$
{ }^{2} B_{2 g} \rightarrow{ }^{2} A_{1 g}:[6 D q-2 D s-6 D t-(-4 D q+2 D s-D t)]=10 D q-4 D s+5 D t
$$

In the above formulae, Dq is octahedral crystal field parameter and Ds, Dt are tetragonal field parameters. The same sign of Dq and Dt indicates an axial elongation and opposite sign indicates an axial compression.

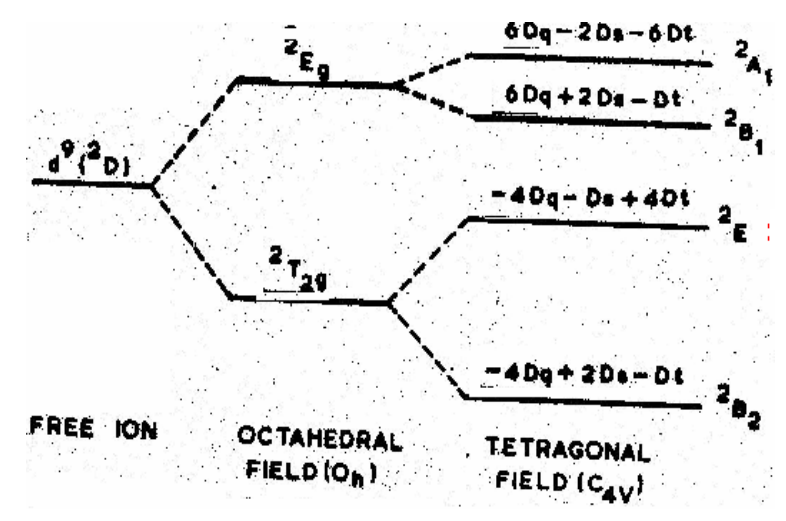

Figure 4. Energy level diagram indicating the assignment of the transitions in octahedral $\mathrm{C}_{4 \mathrm{v}}$ symmetry.

\subsection{EPR spectra of vanadium compounds}

The EPR signal is of three types. (i) is due to high concentration of vanadium. If the vanadium content in the compound is high, it gives a broad resonance line. Therefore the hyperfine line from ${ }^{51} \mathrm{~V}$ cannot be resolved. The $\mathrm{g}$ value for this resonance is less than 2 . (ii) $\mathrm{VO}^{2+}$ ion has $s=1 / 2$ and $\mathrm{I}=7 / 2$. The EPR spectrum shows hyperfine pattern of eight equidistant lines. In $\mathrm{C}_{4 \mathrm{v}}$ symmetry two sets of eight lines are expected (sixteen-line pattern) whereas in $\mathrm{C}_{2 \mathrm{v}}$ symmetry three sets of eight lines are expected. Further in tetragonal distortion, $g_{11}<g_{\perp}<g_{\text {e }}$ which shows the presence of an unpaired electron in the $d_{x y}$ orbital. This is characteristic feature of a tetragonally compressed complex.

Further lowering of symmetry gives rise to EPR spectrum which is similar to the one shown in $\mathrm{g}_{\mathrm{yy}}$ and $\mathrm{g}_{\mathrm{zz}}$ respectively. The hyperfine constants are designated as $\mathrm{A}_{1}, \mathrm{~A}_{2}$ and $\mathrm{A}_{3}$ respectively.

Using the EPR data, the value of dipolar term P and $\mathrm{k}$ term are calculated,

$$
\begin{gathered}
A_{11}=P\left[-\frac{4}{7}-k-\left(g_{11}-g_{e}\right)+\frac{3}{7}\left(g_{\perp}-g_{e}\right)\right] \\
A_{\perp}=P\left[\frac{2}{7}-k+\frac{11}{14}\left[g_{\perp}-g_{e}\right]\right] \\
g=\frac{1}{3}\left(g_{11}+2 g_{\perp}\right) \text { and } A=\frac{1}{3}\left(A_{11}+2 A_{\perp}\right)
\end{gathered}
$$


Using the EPR data, the admixture coefficients are calculated from the following formulae,

$$
\begin{gathered}
g_{11}=2\left(3 C_{1}^{2}-C_{2}^{2}-2 C_{3}^{2}\right) \\
g_{\perp}=4 C_{1}\left(C_{2}-C_{3}\right) \text { and } C_{1}^{2}+C_{2}^{2}+C_{3}^{2}=1 \\
A_{11}=P\left[g_{11}-\left(k+\frac{15}{7}\right)\left(1-2 C_{3}^{2}\right)-\frac{3}{7}\left(1+C_{1} C_{2} C_{3}\right)\right] \\
A_{\perp}=p\left[\frac{11}{14} g_{\perp}-2 C_{1} C_{2}\left(k+\frac{9}{7}\right)\right]
\end{gathered}
$$

\subsection{Relation between EPR and optical absorption spectra}

The optical absorption results and EPR results are related as follows. EPR studies can be correlated with optical data to obtain the orbital coefficients $\beta^{* 2}$ and $\varepsilon_{\pi}^{* 2}$.

$$
\begin{gathered}
g_{11}=g_{e}-\frac{8 \lambda \beta^{* 2}}{\Delta E_{x y}} \\
g_{1}=g_{e}-\frac{2 \lambda \varepsilon_{\pi}^{* 2}}{\Delta E_{x z}}
\end{gathered}
$$

Here $g_{11}$ and $g_{\perp}$ are the spectroscopic splitting factors parallel and perpendicular to the magnetic field direction of $g_{e}$ (i.e., 2.0023 for a free electron).

$\Delta \mathrm{E}_{1}$ is the energy of ${ }^{2} \mathrm{~B}_{2 \mathrm{~g}} \rightarrow{ }^{2} \mathrm{~B}_{1 \mathrm{~g}}$ and $\Delta \mathrm{E}_{2}$ is the energy of ${ }^{2} \mathrm{~B}_{2 \mathrm{~g}} \rightarrow{ }^{2} \mathrm{E}_{\mathrm{g}}$.

$\lambda$ is the spin-orbit coupling constant $\left(160 \mathrm{~cm}^{-1}\right)$ for the free vanadium $\left(\mathrm{VO}^{2+}\right)$.

\subsection{Typical examples}

EPR and optical absorption spectral data of certain selected samples are discussed. The data chosen from the literature are typical for each sample. The data should be considered as representative only. For more complete information on specific example, original references are to be consulted. X-band spectra of the powdered samples and optical absorption spectra are recorded at room temperature (RT).

X-band EPR spectra of the vanadium(IV) complex with DMF recorded in solutions reveal a well-resolved axial anisotropy with 16-line hyperfine structure. This is characteristic of an interaction of vanadium nuclear spin $\left({ }^{51} \mathrm{~V}, I=7 / 2\right)$ with $\mathrm{S}$. The observed EPR parameters are $g_{11}=1.947, A_{11}=161.3 \times 10^{-4} \mathrm{~cm}^{-1}$ and $g \perp=1.978, A \perp=49.0 \times 10^{-4} \mathrm{~cm}^{-1}$. EPR parameters of several samples are available in literature and some of them are given in Table -9 . 


\begin{tabular}{|c|c|c|c|c|}
\hline Mineral name & $g_{11}$ & $g_{\perp}$ & $A_{11} \mathrm{mT}$ & $A_{\perp} \mathrm{mT}$ \\
\hline Kainite & 1.932 & 1.983 & 17.7 & 6.9 \\
Apophyllite & 1.933 & 1.982 & 18.02 & 6.02 \\
Pascoite site I & 1.933 & 1.988 & 18.50 & 7.6 \\
siteII & 1.946 & 1.976 & 20.00 & 8.2 \\
CAPH & 1.933 & 1.993 & & \\
\hline
\end{tabular}

Table 9. Various EPR parameters of VO(II) in minerals

Using the EPR data, the admixture coefficients are calculated for apophyllite and pascoite minerals and are given in the Table -10 .

\begin{tabular}{|lccccc|}
\hline Sample & $\mathrm{C}_{1}$ & $\mathrm{C}_{2}$ & $\mathrm{C}_{3}$ & $K$ & $P\left(\times 10^{-4} \mathrm{~cm}^{-1}\right)$ \\
\hline Apophyllite & 0.7083 & 0.7124 & 0.0028 & 0.86 & 122.7 \\
Pascoite & 0.7010 & 0.7116 & 0.0035 & 0.36 & 118.4 \\
& 0.7090 & 0.7285 & 0.03174 & 0.34 & 143 \\
\hline
\end{tabular}

Table 10. Admixture coefficients of $\mathrm{VO}^{2+}$ ion

EPR spectrum of polycrystalline sample of wavellite with sixteen line pattern indicates the presence of $\mathrm{VO}^{2+}$ ion as an impurity. The EPR parameters calculated are $\mathrm{g}_{z z}=1.933$ and $\mathrm{g}_{\mathrm{yy}}=\mathrm{g}_{\mathrm{xx}}=1.970$ and the corresponding A values are 19.0 and $6.2 \mathrm{mT}$.

\subsection{Typical examples}

a. (i) Divalent vanadium $\left(\mathrm{V}^{2+}\right)$ of $\mathrm{d}^{3}$ configuration, containing halide and other ions in aqueous solutions, gives three transitions, i.e., ${ }^{4} \mathrm{~A}_{2 \mathrm{~g}} \rightarrow{ }^{4} \mathrm{~T}_{2 \mathrm{~g}},{ }^{4} \mathrm{~A}_{2 \mathrm{~g}} \rightarrow{ }^{4} \mathrm{~T}_{1 \mathrm{~g}}(\mathrm{~F})$ and ${ }^{4} \mathrm{~A}_{2 \mathrm{~g}} \rightarrow{ }^{4} \mathrm{~T}_{1 \mathrm{~g}}(\mathrm{P})$ in an octahedral geometry. In $\left[\mathrm{V}\left(\mathrm{H}_{2} \mathrm{O}\right)_{6}^{2+}\right]$, the three bands are observed at 11400, 17100 and $24000 \mathrm{~cm}^{-1}$ along with some weak shoulders at about 20000 and $22000 \mathrm{~cm}^{-1}$. The bands observed at 11400, 17100 and $24000 \mathrm{~cm}^{-1}$ are assigned to the transitions ${ }^{4} \mathrm{~A}_{2 \mathrm{~g}} \rightarrow{ }^{4} \mathrm{~T}_{2 \mathrm{~g}},{ }^{4} \mathrm{~T}_{1 \mathrm{~g}}(\mathrm{~F})$ and ${ }^{4} \mathrm{~T}_{1 \mathrm{~g}}(\mathrm{P})$ respectively. $10 \mathrm{Dq}$ is $11400 \mathrm{~cm}^{-1}$. For divalent vanadium ion, Racah parameters are $\mathrm{B}=860$ and $\mathrm{C}=4165 \mathrm{~cm}^{-1}$. Calculated Racah parameters are expected to be less than the one in the free ion value. Accordingly, the weak shoulders observed at 20000 and $22000 \mathrm{~cm}^{-1}$ are assigned to ${ }^{4} \mathrm{~A}_{2 \mathrm{~g}} \rightarrow{ }^{2} \mathrm{~T}_{2 \mathrm{~g}}$, and ${ }^{4} \mathrm{~A}_{2 \mathrm{~g}} \rightarrow{ }^{2} \mathrm{~T}_{1 \mathrm{~g}}{ }^{2} \mathrm{E}$ transitions.

(ii) The optical absorption spectrum of vanadium carboxylate tetrahydrate sample displays three bands at 11400, 17360 and $23920 \mathrm{~cm}^{-1}$. These are assigned to the transitions, ${ }^{4} \mathrm{~A}_{2 g} \rightarrow{ }^{4} \mathrm{~T}_{2 g},{ }^{4} \mathrm{~T}_{1 g}(\mathrm{~F})$ and ${ }^{4} \mathrm{~T}_{1 \mathrm{~g}}(\mathrm{P})$ in an octahedral geometry. 
b. Trivalent Vanadium $\left(V^{3+}\right)\left(d^{2}\right)$ in aqueous solutions shows two stronger bands at about 15000 and $23000 \mathrm{~cm}^{-1}$ and some weaker bands at 11500, $18000 \mathrm{~cm}^{-1}$. The stronger bands are assigned to the transitions, ${ }^{3} \mathrm{~T}_{1 \mathrm{~g}}(\mathrm{~F}) \rightarrow{ }^{3} \mathrm{~T}_{2 \mathrm{~g}}(\mathrm{~F})$ and ${ }^{3} \mathrm{~T}_{1 \mathrm{~g}}(\mathrm{~F}) \rightarrow{ }^{3} \mathrm{~T}_{1 \mathrm{~g}}(\mathrm{P})$ in an octahedral environment. Since this ion contains two d electrons, it is not so easy to attribute to the other bands. Therefore T-S diagrams are used to identify the other bands. 10Dq is 16400 $\mathrm{cm}^{-1}$ and $\mathrm{B}=623$ (free ion $\mathrm{B}=886 \mathrm{~cm}^{-1}$ and $\mathrm{C}=765 \mathrm{~cm}^{-1}$ ). Third band could be expected at $32000 \mathrm{~cm}^{-1}$ due to ${ }^{3} \mathrm{~T} 1 \mathrm{~g}(\mathrm{~F}) \rightarrow{ }^{2} \mathrm{~A} 1 \mathrm{~g}$. This band corresponds to double electron transition and hence the intensity is expected to be lower than that of the first two bands. The weaker bands observed at 11500, $18000 \mathrm{~cm}^{-1}$ are attributed to the spin forbidden transitions, ${ }^{3} \mathrm{~T}_{1 \mathrm{~g}}(\mathrm{~F}) \rightarrow{ }^{1} \mathrm{E}_{\mathrm{g}},{ }^{1} \mathrm{~T}_{2 \mathrm{~g}}$ and ${ }^{1} \mathrm{~A}_{1 \mathrm{~g}}$.

c. (i) Tetravalent vanadium $\left(\mathrm{V}^{4+}\right)\left(\mathrm{d}^{1}\right)$. The absorption spectrum of tetravalent vanadium compounds shows three transitions, ${ }^{2} \mathrm{~B}_{2 g} \rightarrow{ }^{2} \mathrm{Eg}_{\mathrm{g}}{ }^{2} \mathrm{~B}_{2 \mathrm{~g}} \rightarrow{ }^{2} \mathrm{~B}_{1 \mathrm{~g}}$ and ${ }^{2} \mathrm{~B}_{2 \mathrm{~g}} \rightarrow{ }^{2} \mathrm{~A}_{1 \mathrm{~g}}$. The ${ }^{2} \mathrm{~B}_{2 \mathrm{~g}} \rightarrow{ }^{2} \mathrm{Eg}$ is the most intense and ${ }^{2} \mathrm{~B}_{2 \mathrm{~g}} \rightarrow{ }^{2} \mathrm{~B}_{1 \mathrm{~g}}$ is the weakest. Accordingly, the bands observed in vanadium doped zinc hydrogen maleate tetrahydrate (ZHMT) at 13982, 16125 and $21047 \mathrm{~cm}^{-1}$ are assigned to the above three transitions respectively. The octahedral crystal field parameter, Dq $\left(1613 \mathrm{~cm}^{-1}\right)$, and tetragonal field parameters, Ds $\left(-2700 \mathrm{~cm}^{-1}\right)$ and Dt $\left(1178 \mathrm{~cm}^{-1}\right)$, are evaluated.

(ii) The electronic absorption spectrum of the $\mathrm{VO}^{2+}$ in $\mathrm{CdSO}_{4} .8 \mathrm{H}_{2} \mathrm{O}$ recorded at room temperature shows bands at 12800, 13245, 14815, $18345 \mathrm{~cm}^{-1}$. These bands are assigned to ${ }^{2} \mathrm{~B}_{2 \mathrm{~g}} \rightarrow{ }^{2} \mathrm{E}_{\mathrm{g}},{ }^{2} \mathrm{~B}_{2 \mathrm{~g}} \rightarrow{ }^{2} \mathrm{~B}_{1 \mathrm{~g}}$ and ${ }^{2} \mathrm{~B}_{2 \mathrm{~g}} \rightarrow{ }^{2} \mathrm{~A}_{1 \mathrm{~g}}$ transitions. The band observed at 12500 $\mathrm{cm}^{-1}$ is the split component of the band at $13245 \mathrm{~cm}^{-1}$. The crystal field octahedral parameter, Dq $\left(1465 \mathrm{~cm}^{-1}\right)$ and tetragonal field parameters, Ds $\left(-2290 \mathrm{~cm}^{-1}\right)$ and $\mathrm{Dt}$ $\left(1126 \mathrm{~cm}^{-1}\right)$ are evaluated.

Several examples are found in the literature. Some of them are given in the Table- 11.

\begin{tabular}{|c|c|c|c|c|c|c|}
\hline \multirow{2}{*}{ Sample } & \multicolumn{3}{|c|}{ Transition from ${ }^{2} \mathrm{~B}_{2} \mathrm{~cm}^{-1}$} & \multirow{2}{*}{$\mathrm{Dq} \mathrm{cm} \mathrm{cm}^{-1}$} & \multirow{2}{*}{ Ds cm $\mathrm{cm}^{-1}$} & \multirow{2}{*}{ Dt cm $\mathrm{cm}^{-1}$} \\
\hline & ${ }^{2} \mathrm{E}$, & ${ }^{2} \mathrm{~B}_{1}$ & ${ }^{2} \mathrm{~A}_{1}$ & & & \\
\hline $\begin{array}{l}\text { Cadmium } \\
\text { ammonium } \\
\text { phosphate } \\
\text { hexahydradate(C } \\
\text { APH) }\end{array}$ & 12270 & 16000 & 26625 & 1600 & -3275 & 488 \\
\hline Aphophyllite & 12500 & 15335 & 24385 & 1538 & -2080 & 653 \\
\hline Pascoite site I & 12255 & 14450 & 21415 & 1445 & -2765 & 803 \\
\hline Site II & 12255 & 16000 & 21415 & 1600 & -2524 & 937 \\
\hline
\end{tabular}

Table 11.

d. Pentavalent vanadium has no $\mathrm{d}$ electron and hence $\mathrm{d}-\mathrm{d}$ transitions are not possible. Therefore, the observed bands in electronic absorption spectrum are ascribed to charge transfer bands. These appear around $37000,45000 \mathrm{~cm}^{-1}$. These are assigned to transitions from ligand orbitals to metal d-orbitals: $\mathrm{A}_{1} \rightarrow \mathrm{T}_{2}\left(\mathrm{t}_{1} \rightarrow 2 \mathrm{e}\right)$ and $\mathrm{A}_{1} \rightarrow \mathrm{T}_{2}\left(3 \mathrm{t}_{2} \rightarrow 2 \mathrm{e}\right)$ in tetrahedral configuration for the ion $\mathrm{VO}_{4}^{3-}$. 
Vanadium doped silica gel also shows sharp band at $41520 \mathrm{~cm}^{-1}$ and shoulders at 45450 and $34480 \mathrm{~cm}^{-1}$. These are also assigned to charge transfer transitions in tetrahedral environment of $\mathrm{VO}_{4}^{3-}$. The minimum value of $10 \mathrm{Dq}$ for $\mathrm{VO}_{4}^{3-}$ is expected at about $16000 \mathrm{~cm}^{-1}$ in octahedral geometry. This is expected because the two bands at 34480 and $45450 \mathrm{~cm}^{-1}$ are from the ligand orbitals to two vacant $d$ orbitals which are 10Dq apart. This would be about twice the energy separation $\left(8000 \mathrm{~cm}^{-1}\right)$ observed for tetrahedral $V_{4}^{3-}$. Hence the evidence does not satisfy the assignment of bands to $d-d$ transitions. Therefore the bands are due to charge transfer transitions.

\section{Chromium}

Chromium is the $6^{\text {th }}$ most abundant transition metal. Chromium is used in the manufacture of stainless steel and alloys. The ground state electronic configuration is [Ar] $3 \mathrm{~d}^{4} 4 \mathrm{~s}^{2}$. It exhibits +2 to +6 oxidation states. Most stable oxidation state are $+2(\mathrm{CrO})$, $+3\left(\mathrm{Cr}_{2} \mathrm{O}_{3}\right)$ and $+6\left(\mathrm{~K}_{2} \mathrm{Cr}_{2} \mathrm{O}_{7}\right)$.

\subsection{Optical spectra}

a. Divalent chromium $\left(d^{2}\right)$

$\mathrm{Cr}^{2+}$ has a $\mathrm{d}^{4}$ configuration and forms high spin complexes only for crystal fields less than $2000 \mathrm{~cm}^{-1}$. The ground state term in an octahedral crystal field is ${ }^{5} \mathrm{Eg}$ belonging to the $t_{2 g}^{3} e_{g}^{1}$ configuration. The excited state ${ }^{5} \mathrm{~T}_{2 g}$ corresponds to promotion of one single electron to give $t_{2 g}^{2} e_{g}^{2}$ configuration. The $\mathrm{d}^{4}$ electron is susceptible to Jahn-Teller distortion and hence $\mathrm{Cr}^{2+}$ compounds usually are of low symmetry. In lower symmetry, the excited quintet state of $\mathrm{Cr}^{2+}$ splits into three levels and the ground level quintet state splits into two levels. In the case of $\mathrm{Cr}^{2+}\left(\mathrm{H}_{2} \mathrm{O}\right)_{6}$, the value of $\mathrm{Dq}$ is $1400 \mathrm{~cm}^{-1}$. In spinels, $\mathrm{Cr}^{2+}$ is in the tetrahedral environment and $\mathrm{Dq}$ is about $667 \mathrm{~cm}^{-1}$ only.

b. Trivalent chromium $\left(\mathrm{d}^{3}\right)$ :

In octahedral symmetry, the three unpaired electrons are in $t_{2 g}^{3}$ orbitals which give rise to ${ }^{4} \mathrm{~A}_{2 g},{ }^{2} \mathrm{Eg}_{\mathrm{g}}{ }^{2} \mathrm{~T}_{1 \mathrm{~g}}$ and ${ }^{2} \mathrm{~T}_{2 \mathrm{~g}}$ states. Of these ${ }^{4} \mathrm{~A}_{2 \mathrm{~g}}$ is the ground state. If one electron is excited, the configuration is $t_{2 g}^{2} e_{g}^{1}$ which gives two quartet states ${ }^{4} \mathrm{~T}_{1 g}$ and ${ }^{4} \mathrm{~T}_{2 g}$ and a number of doublet states. When the next electron is also excited, the configuration is $t_{2 g}^{1} e_{g}^{2}$ which gives rise to one quartet state ${ }^{4} \mathrm{~T}_{1 \mathrm{~g}}$ and some doublet states.

$$
\begin{gathered}
{ }^{4} F \rightarrow{ }^{4} A_{2 g}(F),{ }^{4} T_{18}(F),{ }^{4} T_{2 g}(F) \\
{ }^{4} P \rightarrow{ }^{4} T_{18}(P)
\end{gathered}
$$




$$
\begin{gathered}
{ }^{2} G \rightarrow{ }^{2} A_{1 g}(G),{ }^{2} T_{1 g}(G),{ }^{2} T_{2 g}(G),{ }^{2} E_{g}(G) \\
{ }^{2} H \rightarrow{ }^{2} E_{g}(H), 2{ }^{2} T_{1 g}(H),{ }^{2} T_{2 g}(H)
\end{gathered}
$$

In both fields, ${ }^{4} \mathrm{~A}_{2 g}(\mathrm{~F})$ represents the ground state. Hence, three spin allowed transitions are observed in high spin state ${ }^{4} \mathrm{~A}_{2 g}(\mathrm{~F}) \rightarrow{ }^{4} \mathrm{~T}_{2 g}(\mathrm{~F})\left(\mathrm{U}_{1}\right),{ }^{4} \mathrm{~A}_{2 \mathrm{~g}}(\mathrm{~F}) \rightarrow{ }^{4} \mathrm{~T}_{1 \mathrm{~g}}(\mathrm{~F})\left(\mathrm{U}_{2}\right)$ and ${ }^{4} \mathrm{~A}_{2 g}(\mathrm{~F}) \rightarrow{ }^{4} \mathrm{~T}_{1 \mathrm{~g}}(\mathrm{P})(\mathrm{U} 3)$. These spin allowed bands split into two components when the symmetry of $\mathrm{Cr}^{3+}$ ion is lowered from octahedral to $\mathrm{C}_{4 \mathrm{v}}$ or $\mathrm{C}_{3 \mathrm{v}}$. Generally, ${ }^{4} \mathrm{~A}_{2 \mathrm{~g}}(\mathrm{~F}) \rightarrow{ }^{4} \mathrm{~T}_{1 \mathrm{~g}}(\mathrm{P})$ occurs in the UV-Vis region.

The strong field electronic configurations for the ground state and their terms are given as follows:

$$
\begin{gathered}
\left(t_{2 g}\right){ }^{3}\left(e_{g}\right)^{0}:{ }^{4} A_{2 g}(F),{ }^{2} E_{g}(G),{ }^{2} T_{1 g}(G),{ }^{2} T_{2 g}(G) \\
\left(t_{2 g}\right)^{2}\left(e_{g}\right)^{1}:{ }^{4} T_{1 g}(F),{ }^{4} T_{2 g}(F),{ }^{2} T_{2 g}(H) \\
\left(t_{2 g}\right)\left(e_{g}\right)^{2}:{ }^{4} T_{1 g}(P)
\end{gathered}
$$

Racah parameter, B, is calculated with spin allowed transitions using equation (17)

$$
B=\left(2 v_{1}^{2}+v_{2}^{2}-3 v_{1} v_{2}\right) /\left(15 v_{2}-27 v_{1}\right)
$$

The octahedral crystal field parameter $\mathrm{Dq}$ is characteristic of the metal ion and the ligands. The Racah parameter, B depends on the size of the $3 \mathrm{~d}$ orbital; B is inversely proportional to covalency in the crystal.

\subsection{EPR spectra of chromium compounds}

$\mathrm{Cr}^{3+}$ ion, splits into $I \pm 1 / 2>$ and $I \pm 3 / 2>$ Kramers' doublets in the absence of magnetic field, separated by $2 \mathrm{D}$, $\mathrm{D}$ being the zero-field splitting parameter. This degeneracy can be lifted only by an external magnetic field. In such a case, three resonances are observed corresponding to the transitions, $|-3 / 2>\leftrightarrow|-1 / 2>,|-1 / 2>\leftrightarrow| 1 / 2>$ and $|1 / 2>\leftrightarrow| 3 / 2>$ at $g \beta B-2 D, g \beta B$ and $g \beta B+$ $2 \mathrm{D}$ respectively. In a powder spectrum, mainly the perpendicular component is visible. If all the three transitions are observed, the separation between the extreme sets of lines is $4 \mathrm{D}$ [g $\beta B+$ $2 \mathrm{D}-(\mathrm{g} \beta \mathrm{B}-2 \mathrm{D})=4 \mathrm{D}]$. If $\mathrm{D}$ is equal to zero, a single resonance line appears with $\mathrm{g} \sim 1.98$. If $\mathrm{D}$ is very large compared to microwave frequency, a single line is seen around $g=4.0$.

\subsection{Relation between EPR and optical absorption spectra}

A comparison is made between the observed geff from EPR results and the calculated one from the optical spectrum. For $\mathrm{Cr}^{3+}$, EPR and optical results are related by, 


$$
\begin{aligned}
& g_{11}=g_{e}-\frac{8 \lambda}{\Delta E\left({ }^{4} T_{1 g}(F)\right)} \\
& g_{1}=g_{e}-\frac{8 \lambda}{\Delta E\left({ }^{4} T_{2 g}(F)\right)}
\end{aligned}
$$

Here $g_{11}$ and $g_{\perp}$ are the spectroscopic splitting factors parallel and perpendicular to the magnetic field direction, $g$, the free electron value $g_{e}$, is 2.0023 . These values give,

$$
g_{\text {eff }}=\frac{1}{3}\left(g_{11}+g_{1}\right)
$$

The value of $\mathrm{D}$ can also be estimated from the optical absorption spectrum. The ${ }^{4} \mathrm{~A}_{2 g}(\mathrm{~F}) \rightarrow{ }^{4} \mathrm{~T}_{2 g}(\mathrm{~F})$ component in the optical spectrum is due to the lowering of symmetry which also includes the D term.

$$
D=\left(\frac{2 \lambda}{10 D q}\right)^{2}\left(\Delta_{z}-\Delta_{x}\right) .
$$

The spin-orbit splitting parameter, $\lambda$ [for free ion, $\mathrm{Cr}^{3+}$ is $92 \mathrm{~cm}^{-1}$ ] is related to Racah parameter (B) by the equation,

$$
\lambda=0.11(B+1.08)^{2}+0.0062
$$

\subsection{Typical examples}

The data chosen from the literature are typical for each sample. The data should be considered as representative only. For more complete information on specific examples, the original references are to be consulted. X-band spectra and optical absorption spectra of the powdered sample are recorded at room temperature (RT).

1. Trivalent chromium [ $\left.d^{3}\right]$ : The optical absorption spectrum of fuchsite recorded in the mull form at room temperature shows bands at 14925, 15070, 15715, 16400, 17730 and $21740 \mathrm{~cm}^{-1}$. The two broad bands at 16400 and $21740 \mathrm{~cm}^{-1}$ are due to spin-allowed transitions, ${ }^{4} \mathrm{~A}_{2 g}(\mathrm{~F}) \rightarrow{ }^{4} \mathrm{~T}_{2 \mathrm{~g}}(\mathrm{~F})$ and ${ }^{4} \mathrm{~T}_{1 \mathrm{~g}}(\mathrm{~F})$ respectively. The band at $17730 \mathrm{~cm}^{-1}$ is the split component of the ${ }^{4} \mathrm{~T}_{2 \mathrm{~g}}(\mathrm{~F})$ band. This indicates that the site symmetry of $\mathrm{Cr}^{3+}$ is $\mathrm{C}_{4 \mathrm{v}}$ or $\mathrm{C}_{3 \mathrm{v}}$. The bands at 16400 and $21700 \mathrm{~cm}^{-1}$ are responsible for the green color of the mineral. The additional weak features observed for the $v_{1}$ band at 15715 and $15070 \mathrm{~cm}^{-1}$ are attributed to the spin-forbidden transitions, ${ }^{4} \mathrm{~A}_{2 g} \rightarrow{ }^{2} \mathrm{~T}_{1 g}(\mathrm{G})$ and ${ }^{4} \mathrm{~A}_{2 \mathrm{~g}} \rightarrow{ }^{2} \mathrm{Eg}(\mathrm{G})$. Using equation (17), Racah parameter, B, is calculated $\left(507 \mathrm{~cm}^{-1}\right)$. Substituting Dq and B values and using T-S diagrams for $\mathrm{d}^{3}$ configuration and solving the cubic field energy matrices , another Racah parameter, $\mathrm{C}$ is evaluated $\left(2155 \mathrm{~cm}^{-1}\right)$ which is less than the free ion value $\left[\mathrm{C}=3850 \mathrm{~cm}^{-1}\right]$.

Several examples are available in the literature. Some of them are given in the Table- 12 . 


\begin{tabular}{|c|c|c|c|c|c|c|c|c|c|c|c|c|}
\hline Compound & $\begin{array}{c}{ }^{2} \mathrm{Eg}_{\mathrm{g}}(\mathrm{G}) \\
\mathrm{cm}^{-1}\end{array}$ & $\begin{array}{l}{ }^{2} \mathrm{~T}_{1 \mathrm{~g}}(\mathrm{G}) \\
\mathrm{cm}^{-1}\end{array}$ & $\begin{array}{c}{ }^{4} \mathrm{~T}_{2 \mathrm{~g}}(\mathrm{~F}) \\
\left(\mathrm{v}_{1}\right) \\
\mathrm{cm}^{-1}\end{array}$ & $\begin{array}{l}{ }^{2} \mathrm{~T}_{2 \mathrm{~g}}(\mathrm{G}) \\
\mathrm{cm}^{-1}\end{array}$ & $\begin{array}{c}{ }^{4} \mathrm{~T}_{1 \mathrm{~g}}(\mathrm{~F}) \\
\left(\mathrm{v}_{2}\right) \\
\mathrm{cm}^{-1}\end{array}$ & $\begin{array}{c}{ }^{2} \mathrm{~T}_{1 \mathrm{~g}}(\mathrm{H}) \\
\mathrm{cm}^{-1}\end{array}$ & $\begin{array}{c}{ }^{4} \mathrm{~T}_{1 \mathrm{~g}}(\mathrm{P}) \\
\left(\mathrm{v}_{3}\right) \\
\mathrm{cm}^{-1}\end{array}$ & $\begin{array}{c}\mathrm{Dq} \\
\mathrm{cm}^{-1}\end{array}$ & $\begin{array}{c}\mathrm{B} \\
\mathrm{cm}^{-1}\end{array}$ & $\begin{array}{c}\mathrm{C} \\
\mathrm{cm}^{-1}\end{array}$ & $\beta$ & CFSE \\
\hline $\begin{array}{l}\text { Fuchsite } \\
\text { quartz }\end{array}$ & 15500 & 15995 & 19995 & 22720 & 27020 & 35700 & 43465 & 2000 & 677 & 3400 & 0.66 & 24000 \\
\hline Dickite & 14690 & 15500 & 16260 & & 23800 & & 37000 & 1626 & 803 & & 0.78 & 19512 \\
\hline Fuchite & 15070 & 15715 & $\begin{array}{l}16400 \\
17730\end{array}$ & 14925 & 21740 & & & 1640 & 507 & 2155 & 0.49 & 19680 \\
\hline Chromate & & & 17390 & & 23810 & & & 1739 & & & & \\
\hline $\begin{array}{c}\text { Natural } \\
\text { Ruby }\end{array}$ & $\begin{array}{l}14262 \\
14296\end{array}$ & $\begin{array}{l}16725 \\
16919 \\
17042\end{array}$ & $\begin{array}{l}18170 \\
17245\end{array}$ & $\begin{array}{l}21012 \\
21058 \\
21389\end{array}$ & 24993 & & & 1830 & 732 & 2155 & 0.71 & 21960 \\
\hline Variscite & & & $\begin{array}{l}16660 \\
18180\end{array}$ & 15380 & 21735 & 30295 & & 1666 & 475 & 2200 & 0.46 & 19992 \\
\hline $\begin{array}{l}\text { Synthetic } \\
\text { Uvarovite }\end{array}$ & & & 16670 & 18000 & 22730 & & 28000 & & & & & \\
\hline $\begin{array}{c}\mathrm{Sr}_{3} \mathrm{Ga}_{2} \mathrm{Ge}_{4} \mathrm{O}_{14} \\
\text { Garnet }\end{array}$ & & & 16299 & & 433.6 & & & 1629.9 & 712.3 & & 0.69 & 19559 \\
\hline Ureyite & & & 15600 & & 22000 & & & & 664 & & 0.65 & \\
\hline Alexandrite & 14000 & - & 16600 & 21000 & 25000 & & & & & & & \\
\hline Uvarovite & & & 16600 & & 23100 & & & & & & & \\
\hline Clinoclore & & & & & & & & 1834 & 668 & & 0.728 & $63 \times 350$ \\
\hline Amesite & & & & & & & & 1782 & 737 & & 0.899 & $58.0 x$ \\
\hline Muscovite & & & & & & & & 1610 & 737 & & 0.89 & 55.6 \\
\hline Phlogopite & & & & & & & & 1690 & & & & 58.0 \\
\hline
\end{tabular}

1. The EPR spectrum of fuchsite recorded at room temperature (RT) clearly indicates a strong resonance line with a few weak resonances on either side of it. The $\mathrm{g}$ value for this centrally located strong line is 1.98 . This is due to the main transition $|-1 / 2>\leftrightarrow| 1 / 2>$ of $\mathrm{Cr}^{3+}$. The calculated value of $\mathrm{D}$ is around $270 \mathrm{G}$. For weak lines, D is around $160 \mathrm{G}$. Since the lines are equally spaced on either side of the strong resonance, $\mathrm{E}$ is zero. The strong line at $\mathrm{g}(1.98)$ value is observed indicating a high concentration of chromium.

2. The EPR spectrum of chromate shows a broad EPR signal with g value of 1.903 which may be due to $\mathrm{Cr}^{3+}$ which is in high concentration in the mineral. The chromium ion is in octahedral coordination.

3. EPR spectrum of zoisite at LNT givesa $\mathrm{g}$ and $\mathrm{D}$ values of 1.99 and $42.5 \mathrm{mT}$ respectively which are due to $\mathrm{Cr}^{3+}$ in octahedral environment.

4. EPR spectrum of chromium containing fuchsite quartz shows a g value of 1.996 which may due to $\mathrm{Cr}^{3+}$ which is in octahedral environment.

5. EPR spectrum of blue sapphire shows four $\mathrm{Cr}^{3+}$ sites with the same g value of 1.98 having different $\mathrm{D}$ values $(130,105,65$ and $34 \mathrm{mT})$. Green sapphire also has the same $g$ value but different D values $(132,114,94$ and $35 \mathrm{mT})$. The results suggest that chromium content is slightly different in different sapphires.

Table 12. Assignment of bands for $\mathrm{Cr}(\mathrm{III})$ with ${ }^{4} \mathrm{~A}_{2 g}(\mathrm{~F})$ ground state. All values are given in $\mathrm{cm}^{-1}$

Several examples are given in the literature. Some of them are presented in the Table-13.

\begin{tabular}{|l|c|c|c|c|c|c|c|}
\hline \multirow{2}{*}{ Compound } & \multicolumn{3}{|c|}{ Observed } & \multirow{2}{*}{${ }^{4} \mathrm{~T}_{1 \mathrm{~g}}(\mathrm{~F})$} & \multirow{2}{*}{${ }^{4} \mathrm{~T}_{2 \mathrm{~g}}(\mathrm{~F})$} & \multicolumn{2}{c|}{ Calculated } \\
\cline { 2 - 3 } & $g_{\perp}$ & $\mathrm{g}_{11}$ & $\mathrm{~g}_{\text {eff }}$ & $\left(\mathrm{v}_{2}\right) \mathrm{cm}^{-1}$ & $\left(v_{1}\right) \mathrm{cm}^{-1}$ & $\mathrm{~g}_{\text {eff }}$ & $\lambda\left(\mathrm{cm}^{-1}\right.$ \\
\hline Varscite & 1.958 & 1.9684 & 1.994 & 16660 & 21735 & 1.9615 & 75 \\
\hline Chromate & & & 1.903 & & & & \\
\hline
\end{tabular}

Table 13. EPR parameters of $\mathrm{Cr}^{3+}$ compounds. 


\section{Tetravalent chromium $\left(\mathrm{d}^{2}\right)$ :}

Absorption spectra of $\mathrm{Cr}^{4+}$ in forsterite and garnet show the absorption band at $9460 \mathrm{~cm}^{-1}$ which is the typical of $\mathrm{Cr}^{4+}$ ions. It is attributed to the ${ }^{3} \mathrm{~A}_{2 g} \rightarrow{ }^{3} \mathrm{~T}_{2 \mathrm{~g}}$ transition. The absorption band at $19590 \mathrm{~cm}^{-1}$ is also attributed to ${ }^{3} \mathrm{~A}_{2 \mathrm{~g}} \rightarrow{ }^{3} \mathrm{~T}_{1 \mathrm{~g}}$ transition. The absorption band at 19590 $\mathrm{cm}^{-1}$ Overlaps with the bands at 16130 and $23065 \mathrm{~cm}^{-1}$.

\section{Manganese}

The atomic number of manganese is 25 and its outermost electronic configuration is [Ar] $3 \mathrm{~d}^{5} 4 \mathrm{~s}^{2}$. It exhibits several oxidation states, $+2,+3,+4,+6$ and +7 , of which the most stable are $+2+4$ and +7 . The ionic radii of $\mathrm{Mn}^{2+}$ and $\mathrm{Mn}^{4+}$ are 0.80 and 0.54 A.U. respectively. Twenty three isotopes and isomers are known. A number of minerals of manganese exists in nature ( 300 minerals) giving rise to an overall abundance of $0.106 \%$. Twelve of the important among them are economically exploited and the most important of these are pyrolusite $\left(\mathrm{MnO}_{2}\right)$, manganite $\left(\mathrm{Mn}_{2} \mathrm{O}_{3} \cdot \mathrm{H}_{2} \mathrm{O}\right)$, hausmannite $\left(\mathrm{Mn}_{3} \mathrm{O}_{4}\right)$ rhodochrosite $\left(\mathrm{MnCO}_{3}\right)$ and manganese(ocean) nodules. Much of the (85-90\%) manganese is consumed in the manufacture of ferromanganese alloys. The other uses are: manganese coins, dry cell and alkaline batteries and glass. It is an essential trace element for all forms of life.

Octahedral complexes of $\mathrm{Mn}(\mathrm{III})$ are prone to Jahn-Teller distortion. It is of interest, therefore, to compare the structures of $\mathrm{Cr}(\mathrm{acac})_{3}$ with $\mathrm{Mn}(\mathrm{acac})_{3}$ since the former is a regular octahedron while the latter is prone to dynamic Jahn-Teller distortion.

\subsection{EPR spectra of manganese compounds}

1. Manganese(II): Manganese(II), being a $\mathrm{d}^{5}$ ion, is very sensitive to distortions in the presence of magnetic field. $\mathrm{Mn}(\mathrm{II})$ has a total spin, $S=5 / 2$. The six spin states labeled as $\pm 5 / 2>, \pm 3 / 2>$ and $\pm 1 / 2>$ are known as the three Kramers' doublets; in the absence of external magnetic field, they are separated by $4 \mathrm{D}$ and $2 \mathrm{D}$ respectively, where $\mathrm{D}$ is the zero-field splitting parameter. These three doublets split into six energy levels by the application of an external magnetic field. Transitions between these six energy levels give rise to five resonance lines. Each of these resonance lines, in turn, splits into a sextet due to the interaction of the electron spin with the nuclear spin of ${ }^{55} \mathrm{Mn}$, which is $5 / 2$. Thus one expects a 30- line pattern. However, depending on the relative magnitudes of D and A (hyperfine coupling constant of manganese), these 30 lines appear as a separate bunch of 30 lines or 6 lines (if $\mathrm{D}=0$ ). The separation between the extreme set of resonance lines is approximately equal to $8 \mathrm{D}$ (first order). If $\mathrm{D}$ is very small compared to hyperfine coupling constant (A), the 30 lines are so closely packed that one could see only six lines corresponding $-1 / 2$ to $+1 / 2$ transition. If $D=0$, the system is perfectly octahedral. Deviation from axial symmetry leads to a term known as $\mathrm{E}$ in the spin- Hamiltonian. The value of $\mathrm{E}$ can be easily calculated from single crystal measurements. A non-zero value of $\mathrm{E}$ results in making the spectrum unsymmetrical about the central sextet. 
Further, the following parameters have been calculated from the powder spectrum using the Spin- Hamiltonian of the form:

$$
H=\beta 1 B_{3 g} S+D\left(S_{z}^{2}-\frac{1}{3} S(S+1)\right)+S A 1
$$

Here the first term represents the electron-Zeeman interaction, the second term represents the zero field contribution and the third term represents the nuclear-Zeeman interaction. The extra set of resonances within the main sextet is due to the forbidden transitions. From the forbidden doublet lines, the Zero field splitting parameter, D is calculated using the formula,

$$
\begin{gathered}
\Delta H=\left(\frac{2 D^{2}}{H_{m}}\right)\left[\frac{1+16\left(H_{m}-8 A m\right)^{2}}{9 H_{i} H_{m}-64 A m}\right] \\
H_{m}=H_{o}-A m-\frac{\left[I(I+1)-m^{2}\right] A^{2}}{2 H_{o}} \text { or } H_{m}=H_{0}-A m-\left(35-4 m^{2}\right)\left(\frac{A^{2}}{8 H_{0}}\right)
\end{gathered}
$$

where $\mathrm{H}_{\mathrm{m}}$ is the magnetic field corresponding to $\mathrm{m} \leftrightarrow \mathrm{m}$ in HF line; $\mathrm{H}_{0}$ is the resonance magnetic field and $\mathrm{m}$ is the nuclear spin magnetic quantum number.

Percentage of covalency of Mn-ligand bond can be calculated in two ways using (i) Matumura's plot and (ii) electro negativities, $X_{p}$ and $X_{q}$ using the equation,

$$
C=\frac{1}{n}\left[1-0.16\left(X_{p}-X_{q}\right)-0.035\left(X_{p}-X_{q}\right)^{2}\right]
$$

Here $\mathrm{n}$ is the number of ligands around $\mathrm{Mn}(\mathrm{II})$ ion; $\mathrm{X}_{\mathrm{p}}=\mathrm{X}_{\mathrm{Mn}}=1.6$ for $\mathrm{Mn}(\mathrm{II})$ and $\mathrm{X}_{\mathrm{q}}=\mathrm{X}_{\text {ligand }}$.

Also hyperfine constant is related to the covalency by,

$$
A_{\text {iso }}=(2.04 \mathrm{C}-104.5) \times 10^{-4} \mathrm{~cm}^{-1}
$$

Further, the $g$ value for the hyperfine splitting is indicative of the nature of bonding. If the $g$ value shows a negative shift with respect to the free electron $g$ value (2.0023), the bonding is ionic and conversely, if the shift is positive, then the bonding is said to be more covalent in nature.

\subsection{Typical examples}

1. Manganese(II): The EPR spectrum of clinohumite contains a strong sextet at the centre corresponding to the electron spin transition $+1 / 2>$ to $-1 / 2>$. In general, the powder spectrum is characterized by a sextet, corresponding to this transition. The other four transitions corresponding to $\pm 5 / 2>\leftrightarrow \pm 3 / 2>$ and $\pm 3 / 2>\leftrightarrow \pm \pm 1 / 2>$ are not seen due to their 
high anisotropy in D. However, in a few cases only, all the transitions are seen. Moreover, the low field transitions are more intense than the high field transitions. In addition, if $\mathrm{E} \neq$ 0 , the EPR spectrum will not be symmetrical about the central sextet. In clinohumite, the spectrum indicates the presence of at least three types of $\mathrm{Mn}(\mathrm{II})$ impurities in the mineral.

The extra set of resonances within the main sextet is due to the forbidden transitions. From the powder spectrum of the mineral, the following parameters are calculated:

Site I: $\quad g=2.000(1), A=9.15(2) \mathrm{mT}$; and $\mathrm{D}=43.8(1) \mathrm{mT}$.

Site II: $\quad g=2.003(2), A=9.23(2) \mathrm{mT}$; and $\mathrm{D}=44.1(1) \mathrm{mT}$.

Site III: $\mathrm{g}=2.007(1), \mathrm{A}=9.40(2) \mathrm{mT}$; and $\mathrm{D}=44.1(1) \mathrm{mT}$.

This large value of $\mathrm{D}$ indicates a considerable amount of distortion around the central metal ion. Since EPR is highly sensitive to Mn(II) impurity, three such sites are noticed. These two sites have close spin- Hamiltonian parameters. A close look at the EPR spectrum indicates a non-zero value of $\mathrm{E}$, which is very difficult to estimate from the powder spectrum.

2. Pelecypod shell EPR spectrum of powdered sample obtained at room temperature indicates the presence of $\mathrm{Mn}$ (II) and Fe(III) impurities. The spectrum contains a strong sextet at the centre of the spectrum corresponding to the electron spin transition $+1 / 2>$ to $-1 / 2>$. Also, the powder spectrum indicates the presence of, at least, three types of $\mathrm{Mn}(\mathrm{II})$ impurities in the pelecypod shell which is noticed at the sixth hyperfine resonance line. The third Mn(II) site is of very low intensity. The extra set of resonances within the main sextet is due to the forbidden transitions. The variations of intensity are also due to the zero field splitting parameter. From the powder spectrum of the compound, the following parameters are calculated using the spin- Hamiltonian of the form:

$$
H=\beta B g S+D\left[S_{Z}^{2}-S^{(S+1)} / 3+S A I\right]
$$

where the symbols have their usual meaning.

Site I: $\quad g=2.002(1), A=9.33(2) \mathrm{mT}$; and $\mathrm{D}=43.8(1) \mathrm{mT}$

Site II: $\quad g=1.990(2), A=9.41(2) \mathrm{mT}$; and $\mathrm{D}=44.1(1) \mathrm{mT}$

Site III: $g=1.987(1), A=9.49(2) \mathrm{mT}$; and $\mathrm{D}=44.1(1) \mathrm{mT}$

This large value of $\mathrm{D}$ indicates a considerable amount of distortion around the central metal ion. A close look at the EPR spectrum indicates a non-zero value for E.

The hyperfine constant ' $\mathrm{A}$ ' value provides a qualitative measure of the ionic nature of bonding of $\mathrm{Mn}(\mathrm{II})$ ion. The percentage of covalency of Mn-ligand bond is calculated using 'A' $(9.33 \mathrm{mT})$ value obtained from the EPR spectrum and with Matumura's plot. It corresponds to an ionicity of $94 \%$. Also, the approximate value of hyperfine constant (A) is calculated by using the equation (27). 
The value obtained is $92 \times 10^{-4} \mathrm{~cm}^{-1}$. This calculated value agrees well with the observed hyperfine constant $\left(93.3 \times 10^{-4} \mathrm{~cm}^{-1}\right)$ indicating ionic character of $\mathrm{Mn}-\mathrm{O}$ bond in the shell under study.

Using the covalency, the number of ligands around $\mathrm{Mn}(\mathrm{II})$ ion is estimated using the equation (26)

$$
C=\frac{1}{n}\left[1-0.16\left(X_{p}-X_{q}\right)-0.035\left(X_{p}-X_{q}\right)^{2}\right]
$$

Where $X_{P}$ and $X_{q}$ are the electronagativities of metal and ligand. Assuming $X_{p}=X_{M n}=1.4$ and $\mathrm{X}_{\mathrm{q}}=\mathrm{X}_{\mathrm{O}}=3.5$, the number of ligands (n) obtained are 18. This suggests that $\mathrm{Mn}(\mathrm{II})$ may be surrounded by eighteen oxygens of six $\mathrm{CO}_{3}^{2-}$ ions.

i. $\quad$ Manganese (IV): This ion in biological samples gives rise to EPR signal around 3.30.

ii. $\left(\mathrm{Mn}^{7+}\right.$ ion also gives EPR resonance signal at about 2.45 in ceramic materials and in biological samples.

\subsection{Optical absorption studies}

1. Manganese(II): The free ion levels of $\mathrm{Mn}^{2+}$ are ${ }^{6} \mathrm{~S},{ }^{4} \mathrm{G},{ }^{4} \mathrm{P} .{ }^{4} \mathrm{D}$ and ${ }^{4} \mathrm{~F}$ in the order of increasing energy. The energy levels for $\mathrm{Mn}^{2+}$ ion in an octahedral environment are ${ }^{6} \mathrm{~A}_{1 g}(\mathrm{~S}),{ }^{4} \mathrm{~T}_{1 \mathrm{~g}}(\mathrm{G}),{ }^{4} \mathrm{~T}_{2 \mathrm{~g}}(\mathrm{G}),{ }^{4} \mathrm{Eg}(\mathrm{G}),{ }^{4} \mathrm{~T}_{1 \mathrm{~g}}(\mathrm{G}){ }^{4} \mathrm{~A}_{1 \mathrm{~g}},{ }^{4} \mathrm{~T}_{2 \mathrm{~g}}(\mathrm{G}),{ }^{4} \mathrm{E}_{\mathrm{g}}(\mathrm{D}),{ }^{4} \mathrm{~T}_{1 \mathrm{~g}}(\mathrm{P})$ respectively with increasing order of energy. The ${ }^{4} \mathrm{Eg}(\mathrm{G}),{ }^{4} \mathrm{~A} 1 \mathrm{~g}$ and ${ }^{4} \mathrm{Eg}(\mathrm{D})$ levels are less affected when compared to other levels by crystal field. Hence, sharp levels are expected relatively in the absorption spectrum which is the criterion for assignment of levels of $\mathrm{Mn}$ (II) ion. Since all the excited states of $\mathrm{Mn}$ (II) ion will be either be quartets or doublets, the optical absorption spectra of $\mathrm{Mn}$ (II) ions will have only spin forbidden transitions. Therefore, the intensity of transitions is weak.

Energy level diagram of $\mathrm{Mn}$ (II) is extremely complex. Exact solutions for the excited state energy levels in terms of Dq, B and C may be obtained from T-S matrices. These matrices are very large (up to $10 \times 10$ ) and ordinary calculations are not feasible. For this reason, the T-S diagrams given in many places in the literature are not sufficiently complete to allow the assignment of all the observed bands. Therefore a set of computer programmes is written to solve the T-S secular equations for any selected values of B, C and Dq. With the computer program, it is only necessary to obtain values of $B$ and $C$ and the complete scheme for any Dq can be quickly calculated. Fortunately B and C can be obtained analytically, if a sufficiently complete spectrum is obtained using the transitions given below:

$$
\begin{gathered}
{ }^{4} A_{1 g},{ }^{4} E_{g}(G) \rightarrow{ }^{6} A_{1 g}=10 B+5 C=v_{1} \\
{ }^{4} E_{g}(D) \rightarrow{ }^{6} A_{1 g}=17 B+5 C=v_{2}
\end{gathered}
$$


If $v_{1}$ and $v_{2}$ are correctly observed and identified in the spectrum, B and C can be calculated. Identification is particularly easy in these cases because of the sharpness of the bands of these levels and are independent of Dq.

2. Manganese(III): This ion has four $3 \mathrm{~d}$ electrons. The ground state electronic configuration is $t_{2 g}^{3} e_{g}^{1}$. It gives a single spin-allowed transition ${ }^{5} \mathrm{Eg}_{\mathrm{g}} \rightarrow{ }^{5} \mathrm{~T}_{2 g}$ corresponding to one electron transition. This should appear around $20000 \mathrm{~cm}^{-1} \cdot \mathrm{Mn}^{3+}$ cation is subject to Jahn-Teller distortion. The distortion decreases the symmetry of the coordination site from octahedral to tetragonal $\left(\mathrm{D}_{4 \mathrm{~h}}\right)$ or by further lowering the symmetry to rhombic $\left(\mathrm{C}_{2 \mathrm{v}}\right)$. Under the tetragonal distortion, the $\mathrm{t}_{2 \mathrm{~g}}$ orbital splits into $\mathrm{eg}$ and $\mathrm{b}_{2 \mathrm{~g}}$ orbitals whereas the $e_{g}$ orbital splits into $\mathrm{a}_{1 \mathrm{~g}}$ and $\mathrm{b}_{1 \mathrm{~g}}$ orbitals. Hence in a tetragonal site, three absorption bands are observed instead of one. Further distortion splits the eg orbital into singly degenerate $\mathrm{a}_{1 \mathrm{~g}}$ and $\mathrm{b}_{1 \mathrm{~g}}$ orbitals. Thus four bands are observed for rhombic symmetry $\left(\mathrm{C}_{2 \mathrm{v}}\right)$.

The transitions in the tetragonal field are described by the following equations:

$$
\begin{gathered}
{ }^{2} B_{1 g} \rightarrow{ }^{2} A_{1 g}:[6 D q-2 D s-6 D t-(6 D q+2 D s-D t)]=4 D s+5 D t \\
{ }^{2} B_{1 g} \rightarrow{ }^{2} B_{2 g}:[-4 D q-2 D s-D t-(6 D q+2 D s-D t)]=10 D q \\
{ }^{2} B_{1 g} \rightarrow{ }^{2} E_{g}:[-4 D q-D s+4 D t-(6 D q+2 D s-D t)]=10 D q+3 D s
\end{gathered}
$$

In the above equations, Dq is octahedral crystal field and Ds and Dt are tetragonal field parameters. The same sign of $\mathrm{Dq}$ and Dt indicates an axial elongation and opposite sign indicates an axial compression.

The optical absorption bands observed for Mn(III) in octahedral coordination with rhombic distortion $\left(\mathrm{C}_{2 \mathrm{~h}}\right)$ in montmorillonite are given in Table -14 .

\begin{tabular}{|c|c|c|c|c|}
\hline \multicolumn{2}{|c|}{ Assignment } & \multicolumn{3}{|c|}{ Localities } \\
\hline $\mathrm{D}_{4 \mathrm{~h}}$ & $\mathrm{C}_{2 \mathrm{~V}}$ & (Mexico) & (Gumwood Mine) & (California) \\
\hline${ }^{5} \mathrm{~B}_{1 \mathrm{~g}} \rightarrow \mathrm{A}_{1 \mathrm{~g}}$ & ${ }^{5} \mathrm{~B}_{1 \mathrm{~g}}-5 \mathrm{~A}_{1 g}$ & 10480 & 10276 & 10542 \\
${ }^{5} \mathrm{~B}_{1 \mathrm{~g}} \rightarrow \mathrm{B}_{2 \mathrm{~g}}$ & ${ }^{5} \mathrm{~B}_{1 \mathrm{~g}}-5 \mathrm{~A}_{2 \mathrm{~g}}$ & 19041 & 18751 & 18560 \\
& ${ }^{5} \mathrm{~B}_{2 \mathrm{~g}}$ & 20660 & 20496 & 20605 \\
& ${ }^{5} \mathrm{~B}_{1 \mathrm{~g}}-5 \mathrm{~A}_{3 \mathrm{~g}}$ & 21837 & 22127 & 22143 \\
\hline
\end{tabular}

Table 14. Assignment of bands for Mn(III) in montmorillonite

\section{Iron}

The atomic number of iron is 26 and its electronic configuration is [Ar] $4 \mathrm{~s}^{2} 3 \mathrm{~d}^{6}$. Iron has 14 isotopes. Among them, the mass of iron varies from 52 to 60 Pure iron is chemically reactive and corrodes rapidly, especially in moist air or at elevated temperatures. Iron is vital to plant and animal life. The ionic radius of $\mathrm{Fe}^{2+}$ is 0.76 A.U. and that of $\mathrm{Fe}^{3+}$ is 0.64 A.U. The 
most common oxidation states of iron are +2 and +3 . Iron(III) complexes are generally in octahedral in shape, and a very few are in tetrahedral also.

\subsection{EPR spectra of iron compounds}

The EPR spectra of powdered $\mathrm{Fe}^{3+}$ compounds may be described by the spin- Hamiltonian,

$$
H=g B S+D\left(S_{z}^{2}-\frac{1}{3} S(S+1)+E\left(S_{X}^{2}-S_{y}^{2}\right)\right)
$$

The second and third terms in the equation (33) represent the effects of axial and rhombic components of the crystal field respectively. When $\mathrm{D}=\mathrm{E}=0$, it corresponds to a free ion in the magnetic field, $\mathrm{H}$ and if $\mathrm{E}=0$, it implies a field of axial symmetry. If $\lambda(\mathrm{E} / \mathrm{D})$ increases, it results in the variation of rhombic character. Maximum rhombic character is seen at a value of $\lambda=1 / 3$ and further increase in $\lambda$ from $1 / 3$ to 1 results in the decrease of rhombic character. When $\lambda=1$, the axial field situation is reached. When $\lambda=1 / 3$, the $g$ value is around 4.27 and when $\lambda$ is less than $1 / 3$, g value is 4 . Hence, the resonance is no longer isotropic and the powder spectrum in that region is a triplet corresponding to $\mathrm{H}$ along each of the three principle axes. For $\mathrm{Fe}^{3+}$, in fields of high anisotropy, the maximum $\mathrm{g}$ value is 9 . If $\mathrm{g}$ values are limited to 0.80 to 4.30 , the $\mathrm{Fe}^{3+}$ ion is under the influence of a strong tetragonal distortion.

1. Iron (III): The iron (III) samples exhibit a series of $g$ values ranging from 0 to 9 . This is due to the fact that the three Kramers' doublets of $\mid S=5 / 2>$ are split into $|S \pm 5 / 2>|, S \pm 3 / 2>$ and $\mid S \pm 1 / 2>$ separated by $4 \mathrm{D}$ and $2 \mathrm{D}$ respectively where $\mathrm{D}$ is the zero field splitting parameter. Depending on the relative populations of these doublets, one observes $g$ value ranging from 0 to 9.0. The line widths are larger in low magnetic field when compared to high magnetic field. If the lowest doublet, $\mid S \pm 1 / 2>$ is populated, it gives a $g$ value of 2 to 6 whereas if the middle Kramers' doublet $\mid S \pm 3 / 2>$ is populated, a g value 4.30 is expected. If the third doublet $\mid S \pm 5 / 2>$ is populated, it gives a $g$ value of $2 / 7$ to $30 / 7$. A few systems are known which exhibit resonances from all the three Kramers' doublets.

The iron(III) in the natural sample enters the lattice in various locations which may not correspond to the lowest energy configuration. After heating the sample, the impurity settles in the lowest energy configuration and the EPR spectrum is simplified. Thus, it is observed that heating the sample results in a simplification of the EPR spectrum and gives a $\mathrm{g}$ value of around 2 .

\subsection{Typical examples}

1. The EPR spectrum of powdered red sandal wood obtained at room temperature contains a series of lines of various intensity and width. The g values obtained for these are $6.52,2.63$ and 1.92. These three peaks are attributed to Fe(III) impurity in the compound.

2. The EPR spectrum of prehnite at room temperature consists of two parts. The first part consists of the two strong lines (absorption and dispersion) and the second part 
comprises a weak doublet within the strong doublet. The weak doublet also consists of two lines, absorption and dispersion line shapes. The $g$ values of the strong doublet are 4.48 and 3.78 whereas the $g$ values of the weak doublet are 4.22 and 3.96. The data reveal that there are two different centres of Fe(III) which are magnetically distinct.

3. The EPR spectrum of nano iron oxalate recorded at room temperature reveals three sets of four lines in low, medium and high fields corresponding to $g_{1}, g_{2}$ and $g_{3}$ respectively. From the positions of the peaks in the EPR spectrum, the following spectroscopic splitting factors are evaluated: $\mathrm{g}_{1}=2.130, \mathrm{~g}_{2}=2.026$ and $\mathrm{g}_{3}=1.947$. The hyperfine structure constants are $\mathrm{A}_{1}=78 \mathrm{mT}, \mathrm{A}_{2}=46 \mathrm{mT}$ and $\mathrm{A}_{3}=26 \mathrm{mT}$. The EPR spectrum is characteristic of $\mathrm{Fe}(\mathrm{III})$ ion or $\mathrm{HCO}_{2}^{-}$or in rhombic symmetry. For the rhombic symmetry, $g$ values follow in the sequence as $\mathrm{g}_{1}>\mathrm{g}_{2}>\mathrm{g}_{3}$. Using the relation, spin-orbit coupling constant, $\lambda$ is calculated. Resonant value of the magnetic field is given by the relation,

$$
H_{R}(m T)=\frac{21419.49}{g \lambda(\mathrm{cm})}=\frac{0.07144775}{g} v(\mathrm{MHz})
$$

$\lambda$ calculated for each $\mathrm{g}$ tensor is 32.18 .

For axial symmetry, $\lambda$ is zero. If rhombic character in the crystal field is increased, it results in the increase of $\lambda$ upto a maximum of $\frac{1}{3}$. In the present case, the observed $\lambda$ is $\frac{1}{3}(32.18 \%)$. Thus the EPR studies indicate that the iron oxalate nano-crystal is in orthorhombic structure.

\subsection{Optical absorption spectra of iron compounds}

\subsubsection{Trivalent iron}

Trivalent iron has the electronic configuration of $3 d^{5}$ which corresponds to a half-filled $d$ sub-shell and is particularly most stable. In crystalline fields, the usual high spin configuration is $t_{2 g}^{3} e_{g}^{2}$ with one unpaired electron in each of the orbitals and the low spin state has the $t_{2 g}^{5}$ configuration with two pairs of paired electrons and one unpaired electron. The energy level in the crystal field is characterized by the following features. i) The ground state of $\mathrm{d}^{5}$ ion, ${ }^{6} \mathrm{~S}$ transforms into ${ }^{6} \mathrm{~A} 1 \mathrm{~g}$ - a singlet state. It is not split by the effect of crystal field and hence all the transitions are spin forbidden and are of less intensity. ii) In excited state, $d^{5}$ ion gives rise to quartets $\left({ }^{4} \mathrm{G},{ }^{4} \mathrm{~F},{ }^{4} \mathrm{D},{ }^{4} \mathrm{P}\right)$ and doublets $\left({ }^{2} \mathrm{I},{ }^{2} \mathrm{H}\right.$, $\left.{ }^{2} \mathrm{G},{ }^{2} \mathrm{~F},{ }^{2} \mathrm{D},{ }^{2} \mathrm{P},{ }^{2} \mathrm{~S}\right)$. The transitions from the ground to doublet state are forbidden because the spin multiplicity changes by two and hence they are too weak. Thus sextet-quartet forbidden transitions observed are: ${ }^{6} \mathrm{~A}_{1 \mathrm{~g}} \rightarrow{ }^{4} \mathrm{~T}_{1 \mathrm{~g}}$ and ${ }^{6} \mathrm{~A}_{1 \mathrm{~g}} \rightarrow{ }^{4} \mathrm{~T}_{2 \mathrm{~g}}$. The transitions which are independent of Dq and which result in sharp bands are ${ }^{6} \mathrm{~A}_{1 \mathrm{~g}} \rightarrow{ }^{4} \mathrm{E}\left({ }^{4} \mathrm{D}\right){ }^{6} \mathrm{~A}_{1 \mathrm{~g}} \rightarrow{ }^{4} \mathrm{Eg}_{\mathrm{g}}+{ }^{4} \mathrm{E}_{1 \mathrm{~g}}$ etc., iii) The unsplit ground state term behaves alike in both octahedral and tetrahedral symmetries and gives rise to same energy level for octahedral, tetrahedral and cubic coordination with usual difference, 


$$
\left[D q_{\text {Octa }}: D q_{\text {Tetra }}: D q_{\text {Cubic }}=1: \frac{4}{9}: \frac{8}{9}\right]
$$

\subsubsection{Divalent iron}

In divalent iron $\left(\mathrm{d}^{6}\right)$, the free ion ground term is ${ }^{5} \mathrm{D}$ and the excited terms are triplet states $\left({ }^{3} \mathrm{H},{ }^{3} \mathrm{P},{ }^{3} \mathrm{~F},{ }^{3} \mathrm{G},{ }^{3} \mathrm{D}\right)$ and singlet states $\left({ }^{1} \mathrm{I},{ }^{1} \mathrm{D}\right)$. In an octahedral field, the ${ }^{5} \mathrm{D}$ term splits into an upper ${ }^{5} \mathrm{Eg}$ level and a lower ${ }^{5} \mathrm{~T}_{2 \mathrm{~g}}$ level of which the latter forms the ground state. The only allowed transition is ${ }^{5} \mathrm{~T}_{2 \mathrm{~g}} \rightarrow{ }^{5} \mathrm{Eg}$ which gives an intense broad absorption band. This band splits into two bands due to Jahn-Teller effect. The average of these two bands is to be taken as 10Dq band. The transitions arising from the excited triplet states are spin forbidden and hence are weaker than the $10 \mathrm{Dq}$ band.

\subsubsection{Typical examples}

1. For $\mathrm{Fe}^{3+}$, there are three transitions: ${ }^{6} \mathrm{~A}_{1 \mathrm{~g}}(\mathrm{~S}) \rightarrow{ }^{4} \mathrm{~T}_{1 \mathrm{~g}}(\mathrm{G})\left(\mathrm{v}_{1}\right),{ }^{6} \mathrm{~A}_{1 \mathrm{~g}}(\mathrm{~S}) \rightarrow{ }^{4} \mathrm{~T}_{2 g}(\mathrm{G})\left(\mathrm{v}_{2}\right)$. $v_{1}$ occurs between $10525 \mathrm{~cm}^{-1}$ and $v_{2}$ occurs between 15380 to $18180 \mathrm{~cm}^{-1}$ usually as a shoulder. The bands corresponding to ${ }^{6} \mathrm{~A}_{1 g}(\mathrm{~S}) \rightarrow{ }^{4} \mathrm{~A}_{1 \mathrm{~g}}(\mathrm{G}),{ }^{4} \mathrm{E}_{\mathrm{g}}(\mathrm{G})\left(\mathrm{v}_{3}\right)$ appear around $22000 \mathrm{~cm}^{-1}$. The last transition is field independent. The ligand field spectrum of ferric iron appears as if the first $\left(v_{1}\right)$ and the third $\left(v_{3}\right)$ bands of octahedral symmetry are only present. The analysis of general features of the spectrum of $\mathrm{Fe}^{3+}$ containing plumbojarosite is discussed here. The first feature observed in the range 12000 to $15500 \mathrm{~cm}^{-1}$ is attributed to ${ }^{6} \mathrm{~A}_{1 \mathrm{~g}}(\mathrm{~S}) \rightarrow{ }^{4} \mathrm{~T}_{1 \mathrm{~g}}(\mathrm{G})$, the third band at $22730 \mathrm{~cm}^{-1}$ is sharp and is assigned to ${ }^{6} \mathrm{~A} 1 \mathrm{~g}(\mathrm{~S}) \rightarrow$ ${ }^{4} \mathrm{~A}_{1 \mathrm{~g}}(\mathrm{G}),{ }^{4} \mathrm{E}_{\mathrm{g}}(\mathrm{G})$ transitions respectively. A broad and diffused band at $19045 \mathrm{~cm}^{-1}$ is assigned to the ${ }^{6} \mathrm{~A}_{1 \mathrm{~g}}(\mathrm{~S}) \rightarrow{ }^{4} \mathrm{~T}_{2 \mathrm{~g}}(\mathrm{G})$ band. The other bands are also assigned to the transitions with the help of Tanabe-Sugano diagram. The assignments are given in the Table -15 .

2. Optical absorption spectrum of prehnite recorded in the mull form at room temperature (RT) shows bands at 9660, 10715, 12100, 12610, 15270, 16445,17095, 23380 and $24390 \mathrm{~cm}^{-1}$ in the UV -Vis region. For easy analysis of the spectrum, the bands are divided into two sets as 12100, 15270, $23380 \mathrm{~cm}^{-1}$ and 12610, 16445, 17095, $24390 \mathrm{~cm}^{-1}$. Accordingly the two bands observed at $12100 \mathrm{~cm}^{-1}$ in the first set and $12610 \mathrm{~cm}^{-1}$ in the second set are assigned to the same transition ${ }^{6} \mathrm{~A}_{1 \mathrm{~g}}(\mathrm{~S}) \rightarrow{ }^{4} \mathrm{~T}_{1 \mathrm{~g}}(\mathrm{G})$ whereas $15270 \mathrm{~cm}^{-1}$ in the first set and $16445,17095 \mathrm{~cm}^{-1}$ in the second set are assigned to ${ }^{4} \mathrm{~T}_{2 \mathrm{~g}}(\mathrm{G})$ transition. The third at $23380 \mathrm{~cm}^{-1}$ and $24390 \mathrm{~cm}^{-1}$ is assigned to ${ }^{4} \mathrm{~A}_{1 \mathrm{~g}}(\mathrm{G}),{ }^{4} \mathrm{E}(\mathrm{G})$ ( $\mathrm{v}_{3}$ ) transitions respectively. These two sets of bands are characteristic of Fe(III) ion occupying two different sites in octahedral symmetry. The broad and intense band observed at 10715 $\mathrm{cm}^{-1}$ with a split component at $9660 \mathrm{~cm}^{-1}$ is assigned to the transition ${ }^{5} \mathrm{~T}_{2 \mathrm{~g}} \rightarrow{ }^{5} \mathrm{Eg}$ for divalent iron in the sample. Using the Tree's polarization term, $\alpha=90 \mathrm{~cm}^{-1}$, the energy matrices of the $\mathrm{d}^{5}$ configuration are solved for various $\mathrm{B}, \mathrm{C}$ and $\mathrm{Dq}$ values. The evaluated parameters which give good fit are given in Table 15. A comparison is also made between the calculated and observed energies of the bands and these are presented in Table - 15. 


\begin{tabular}{|c|c|c|c|c|c|c|c|c|c|}
\hline \multicolumn{6}{|c|}{ Prehnite } & \multirow{2}{*}{\multicolumn{3}{|c|}{ Plumbojarosite }} & \multirow{5}{*}{$\begin{array}{l}\text { Transition } \\
\text { from }{ }^{6} \mathrm{~A}_{1 g}\end{array}$} \\
\hline \multicolumn{3}{|c|}{ Site I } & \multicolumn{3}{|c|}{ Site II } & & & & \\
\hline \multicolumn{3}{|c|}{$\begin{array}{c}\mathrm{Dq}=930, \mathrm{~B}=600 \text { and } \\
\mathrm{C}=2475 \mathrm{~cm}^{-1}, \alpha=90 \mathrm{~cm}^{-1}\end{array}$} & \multicolumn{3}{|c|}{$\begin{array}{c}\mathrm{Dq}=900, \mathrm{~B}=600 \text { and } \\
\mathrm{C}=2500 \mathrm{~cm}^{-1} \alpha=90 \mathrm{~cm}^{-1}\end{array}$} & \multicolumn{3}{|c|}{$\begin{array}{c}\mathrm{Dq}=900, \mathrm{~B}=700 \text { and } \mathrm{C}=2800 \\
\mathrm{~cm}^{-1} \alpha=90 \mathrm{~cm}^{-1}\end{array}$} & \\
\hline \multirow{2}{*}{$\begin{array}{c}\text { Wave } \\
\text { length } \\
(\mathrm{nm})\end{array}$} & \multicolumn{2}{|c|}{ Wave number $\left(\mathrm{cm}^{-1}\right)$} & \multirow{2}{*}{$\begin{array}{c}\text { Wave } \\
\text { length } \\
(\mathrm{nm})\end{array}$} & \multicolumn{2}{|c|}{ Wave number $\left(\mathrm{cm}^{-1}\right)$} & \multirow{2}{*}{$\begin{array}{c}\text { Wave } \\
\text { length } \\
(\mathrm{nm})\end{array}$} & \multicolumn{2}{|c|}{ Wave number $\left(\mathrm{cm}^{-1}\right)$} & \\
\hline & Observed & Calculated & & Observed & Calculated & & Observed & Calculated & \\
\hline 827 & 12100 & 793 & 793 & 12610 & 12528 & $\begin{array}{c}800650 \\
525\end{array}$ & $\begin{array}{l}12500 \\
15385\end{array}$ & \begin{tabular}{|c|}
-15194 \\
19379
\end{tabular} & ${ }^{4} \mathrm{~T}_{1 \mathrm{~g}}(\mathrm{G})$ \\
\hline \multirow{7}{*}{$\begin{array}{l}655 \\
430\end{array}$} & 15270 & 608 & 608 & 16445 & 16331 & 440 & 19045 & 22766 & ${ }^{4} \mathrm{~T}_{2 \mathrm{~g}}(\mathrm{G})$ \\
\hline & & 585 & 585 & 17095 & & 410 & 22730 & & \\
\hline & 23380 & 427 & 427 & 24390 & 23276 & 385 & & 24815 & ${ }^{4} \mathrm{~A} 1 \mathrm{~g}(\mathrm{G})$ \\
\hline & & & & & & 330 & 24390 & 26474 & ${ }^{4} \mathrm{E}(\mathrm{G})$ \\
\hline & & & & & & $\begin{array}{l}263 \\
240\end{array}$ & $\begin{array}{l}259 / 5 \\
30300\end{array}$ & $\begin{array}{l}30656 \\
37710\end{array}$ & ${ }^{4} \mathrm{~T}_{2 \mathrm{~g}}(\mathrm{D})$ \\
\hline & & & & & & & 37735 & 41125 & \\
\hline & & & & & & & 41665 & & ${ }^{4} \mathrm{Eg}_{\mathrm{g}}(\mathrm{D})$ \\
\hline
\end{tabular}

Table 15. Band headed data with assignments for Fe(III) in various compounds

\section{Nickel}

Nickel is the $7^{\text {th }}$ most abundant transition metal in the earth's crust. The electronic configuration of nickel is [Ar] $4 \mathrm{~S}^{2} 3 \mathrm{~d}^{8}$. Nickel occurs in nature as oxide, silicate and sulphide. The typical examples are garnierite and pentlandite. Nickel exhibits +1 to +4 oxidation states. Among them divalent state is most stable. Nickel compounds are generally blue and green in color and are often hydrated. Further, most nickel halides are yellow in color. The primary use of nickel is in the preparation of stainless steel. Nickel is also used in the coloring of glass to which it gives a green hue.

\subsection{Electronic spectra of nickel compounds}

The electronic distribution of $\mathrm{Ni}(\mathrm{II})$ ion $\left(\mathrm{d}^{8}\right)$ is $t_{2 g}^{6} e_{g}^{2}$ which gives rise to ${ }^{3} \mathrm{~F},{ }^{3} \mathrm{P},{ }^{1} \mathrm{D}$ and ${ }^{1} \mathrm{~S}$ terms of which ${ }^{3} \mathrm{~F}$ is the ground state. In a cubic crystal, these terms transform as follows:

$$
\begin{gathered}
{ }^{3} F \rightarrow{ }^{3} T_{1 g}(F)+{ }^{3} T_{2 g}(F)+{ }^{3} A_{2 g}(F) \\
{ }^{3} F \rightarrow{ }^{3} T_{1 g}(P) \\
{ }^{1} D \rightarrow{ }^{3} T_{2 g}(D)+{ }^{1} E_{g}(D) \\
{ }^{1} G \rightarrow{ }^{1} T_{1 g}(G)+{ }^{1} T_{2 g}(G)+{ }^{1} E_{g}(G)+{ }^{1} A_{1 g}(G) \\
{ }^{1} S \rightarrow{ }^{1} A_{1 g}(S)
\end{gathered}
$$


Of these crystal field terms, ${ }^{3} \mathrm{~A}_{2 g}(\mathrm{~F})$ is the ground state. Hence three spin allowed transitions are possible and the others are spin forbidden The three spin allowed transitions are: ${ }^{3} \mathrm{~A} 2 g(\mathrm{~F})$ $\rightarrow{ }^{3} \mathrm{~T}_{1 g}(\mathrm{P}),{ }^{3} \mathrm{~A}_{2 g}(\mathrm{~F}) \rightarrow{ }^{3} \mathrm{~T}_{1 g}(\mathrm{~F})$ and ${ }^{3} \mathrm{~A}_{2 g}(\mathrm{~F}) \rightarrow{ }^{3} \mathrm{~T}_{2 g}(\mathrm{~F})$. These transitions are governed by linear equations as given below:

$$
\begin{gathered}
{ }^{3} A_{2 g}(F) \rightarrow{ }^{3} T_{1 g}(P)=15 D q+7.5 B+6 B(1+\mu)^{1 / 2}=v_{1} \\
{ }^{3} A_{2 g}(F) \rightarrow{ }^{3} T_{1 g}(F)=15 D q+7.5 B-6 B(1+\mu)^{1 / 2}=v_{2} \\
{ }^{3} A_{2 g}(F) \rightarrow{ }^{3} T_{2 g}(F)=10 D q=v_{3}
\end{gathered}
$$

Here $\mu$ is of the order of $0.01 . \mathrm{Dq}$ and $\mathrm{B}$ are of similar magnitude. The spin allowed bands are calculated using the above equations whereas the spin forbidden bands are assigned using Tanabe-Sugano diagrams.

\subsection{Typical examples}

The data chosen from the literature are typical and representative for each sample. For more complete information on any specific case, original references are to be consulted. X-band spectra and optical absorption spectra of the powdered sample are recorded at room temperature (RT) only.

Divalent Nickel $\left[d^{8}\right]$ : The optical absorption spectrum of falcondoite mineral recorded in the mull form at room temperature shows three intense bands at 9255,15380 and $27390 \mathrm{~cm}^{-1}$ and a weak band at $24385 \mathrm{~cm}^{-1}$. Using the equations 34 to 36 , the calculated values of $\mathrm{Dq}$ and $\mathrm{B}$ are 925 and $1000 \mathrm{~cm}^{-1}$ respectively. Using these Dq and B values and T-S diagrams for $\mathrm{d}^{8}$ configuration, the cubic field energy matrices and Racah parameter, $C$ are evaluated (4.1B) .

$\mathrm{Ni}^{2+}$ also gives absorption bands in the NIR region. These bands suggest that $\mathrm{Ni}^{2+}$ is in

\begin{tabular}{|c|c|c|c|c|c|c|c|c|c|c|c|}
\hline Compound & $\begin{array}{c}{ }^{3} \mathrm{~T}_{\mathrm{g}}(\mathrm{P}) \\
\left(\mathrm{v}_{1}\right)\end{array}$ & $\begin{array}{c}{ }^{3} \mathrm{~T}_{1 g}(\mathrm{~F}) \\
\left(\mathrm{V}_{2}\right)\end{array}$ & $\begin{array}{c}{ }^{3} \mathrm{~T}_{2 g}(\mathrm{~F}) \\
\left(v_{3}\right)\end{array}$ & ${ }^{1} \mathrm{~T}_{1 \mathrm{~g}}(\mathrm{G})$ & ${ }^{1} \mathrm{~T}_{1} \mathrm{~g}(\mathrm{D})$ & ${ }^{1} \mathrm{Eg}(\mathrm{D})$ & ${ }^{1} \mathrm{~T}_{2 \mathrm{~g}}(\mathrm{D})$ & ${ }^{1} \mathrm{~T}_{2 \mathrm{~g}}(\mathrm{G})$ & $\mathrm{Dq}$ & B & $\mathrm{C}$ \\
\hline Falcondoite & 27390 & 15380 & 9255 & & & & 24385 & & 925 & 1000 & 4100 \\
\hline Ullmannite & 24993 & 14966 & 8618 & 25967 & 21546 & 12252 & 21546 & & 860 & 840 & 3350 \\
\hline Takovite & 26665 & 15380 & $\begin{array}{c}8200 \\
10000 \\
\end{array}$ & & & 14930 & 24095 & & 910 & 940 & $4.25 \mathrm{~B}$ \\
\hline $\begin{array}{l}(\mathrm{Zn}, \mathrm{Ni}) \mathrm{KPO} \\
{ }_{46} \mathrm{H}_{2} \mathrm{O}\end{array}$ & 25967 & 15500 & 8770 & & & 14080 & 22216 & & 900 & 890 & 3800 \\
\hline \multicolumn{12}{|l|}{ Ni(II) HZDT } \\
\hline Garnierite & 26300 & 15200 & 9100 & & & 13000 & & & & & \\
\hline Gaspeite & 22730 & $\begin{array}{l}13160 \\
14705 \\
\end{array}$ & $\begin{array}{l}7714 \\
8685 \\
\end{array}$ & & & & 20410 & 30300 & 810 & 800 & 3200 \\
\hline Annabergite & & 13885 & 8330 & & & & & & & & \\
\hline Zartite & 23805 & 14285 & 8195 & & & & 21735 & & 820 & 899 & $4.1 \mathrm{~B}$ \\
\hline
\end{tabular}
tetrahedral site. In some of the samples, $\mathrm{Ni}^{2+}$ exbits both octahedral and tetrahedral coordination. Several examples are available in the literature. Some of them are given in the Table-16.

Table 16. Assignment of bands for $\mathrm{Ni}(\mathrm{II})$ with ${ }^{3} \mathrm{~A} 2 \mathrm{~g}(\mathrm{~F})$ as the ground state. All values are given in $\mathrm{cm}^{-1}$. 


\subsection{EPR spectra}

$\mathrm{Ni}^{2+}\left(\mathrm{d}^{8}\right)$ has no unpaired electron (square planer) in its orbit. Therefore it does not exhibit EPR signal at room temperature.

But in certain conditions, it shows EPR signal. The EPR data could be related with the optical data by the following equation $g=2.0023-\frac{8 \lambda}{\Delta}$ where $\Delta$ is the energy of the transition of the perfect octahedral site. $\lambda$ is $324 \mathrm{~cm}^{-1}$ for free $\mathrm{Ni}^{2+}$ ion.

\section{Copper}

Copper is one of the earliest known elements to man. The average percentage of copper in the earth's crust is $0.005 \%$. Pure copper is soft and malleable. An important physical property of copper is its color. Most people refer copper colour as reddish-brown tint. Copper-63 and copper-65 are two naturally occurring isotopes of copper. Nine radioactive isotopes of copper are also known. Among them two radioactive isotopes, copper-64 and copper-67 are used in medicine. Copper easily reacts with oxygen and in moist air, it combines with water and carbon dioxide forming hydroxy copper carbonate $\left(\mathrm{Cu}_{2}(\mathrm{OH})_{2} \mathrm{CO}_{3}\right)$.

Animals like crustaceans (shellfish like lobsters, shrimps, and crabs) do not have hemoglobin to carry oxygen through the blood but possess a compound called hemocyanin. This is similar to hemoglobin but contains copper instead of iron. Copper is an essential micronutrient for both plants and animals. A healthy human requires not more than about 2 mg of copper for every $\mathrm{kg}$ weight of the body. The main body parts where copper is found in animals are the tissues, liver, muscle and bone.

\subsection{Copper compounds}

Copper exists in two ionic states, $\mathrm{Cu}(\mathrm{I})$ and $\mathrm{Cu}(\mathrm{II})$. The ionic radius of $\mathrm{Cu}(\mathrm{II})$ is 0.73 A.U. The electronic configuration of $\mathrm{Cu}(\mathrm{I})$ is $[\mathrm{Ar}] 3 \mathrm{~d}^{10}$ and hence has no unpaired electron in its outermost orbit. Hence it exhibits diamagnetism. The electronic configuration of $\mathrm{Cu}$ (II) is $[\mathrm{Ar}] 3 \mathrm{~d}^{9}$ and has one unpaired electron which is responsible for its para magnetism. The main resources of copper are its minerals. Structural properties could be explored using electronic and EPR spectra which provides information on bonding between ligands and metal ion.

\subsection{Electronic spectra of copper compounds}

In optical spectroscopy, transitions proceed between the split orbital levels whereas in EPR spectroscopy they occur between spin sub- levels that arise due to the external magnetic field. Thus EPR spectroscopy is a natural sequel to optical spectroscopy.

\subsection{Optical spectra}

In octahedral crystal field, the ground state electronic distribution of $\mathrm{Cu}^{2+}$ is $\mathrm{t}_{2 g^{6}} \mathrm{eg}^{3}$ which yields ${ }^{2} \mathrm{Eg}$ term. The excited electronic state is $\mathrm{t}_{2 \mathrm{~g}}{ }^{5} \mathrm{eg}^{4}$ which corresponds to ${ }^{2} \mathrm{~T}_{2 \mathrm{~g}}$ term. Thus 
only one single electron transition, i.e., ${ }^{2} \mathrm{Eg}_{\mathrm{g}} \rightarrow{ }^{2} \mathrm{~T}_{2 \mathrm{~g}}$, is expected in an octahedral crystal field. The difference is 10Dq. Octahedral coordination is distorted either by elongation or compression of octahedron leading to tetragonal symmetry.

Normally, the ground ${ }^{2} \mathrm{Eg}$ state is split due to Jahn-Teller effect and hence lowering of

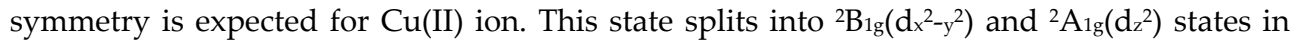
tetragonal symmetry and the excited term ${ }^{2} \mathrm{~T}_{2 g}$ also splits into ${ }^{2} \mathrm{~B}_{2 \mathrm{~g}}\left(\mathrm{~d}_{\mathrm{xy}}\right)$ and ${ }^{2} \mathrm{Eg}_{\mathrm{g}}\left(\mathrm{d}_{\mathrm{xz}}, \mathrm{d}_{\mathrm{yz}}\right)$ levels. In rhombic field, ${ }^{2} \mathrm{Eg}$ ground state is split into ${ }^{2} \mathrm{~A}_{1 \mathrm{~g}}\left(\mathrm{dx}^{2}-\mathrm{y}^{2}\right)$ and ${ }^{2} \mathrm{~A}_{2 \mathrm{~g}}\left(\mathrm{dz}^{2}\right)$ whereas ${ }^{2} \mathrm{~T}_{2 \mathrm{~g}}$ splits into ${ }^{2} \mathrm{~B}_{1 \mathrm{~g}}\left(\mathrm{~d}_{\mathrm{x} y}\right),{ }^{2} \mathrm{~B}_{2 \mathrm{~g}}\left(\mathrm{~d}_{\mathrm{xz}}\right)$ and ${ }^{2} \mathrm{~B}_{3 \mathrm{~g}}\left(\mathrm{~d}_{\mathrm{yz}}\right)$ states. Thus, three bands are expected for tetragonal $\left(\mathrm{C}_{4 \mathrm{v}}\right)$ symmetry and four bands are expected for rhombic $\left(D_{2 h}\right)$ symmetry. Energy level diagram of d-orbitals in tetragonal elongated environment is shown in Fig. 5.

The transitions in the tetragonal field are described by the following equations:

$$
\begin{gathered}
{ }^{2} B_{1 g} \rightarrow{ }^{2} A_{1 g}:[6 D q-2 D s-6 D t-(6 D q+2 D s-D t)]=4 D s+5 D t \\
{ }^{2} B_{1 g} \rightarrow{ }^{2} B_{2 g}:[-4 D q+2 D s-D t-(6 D q+2 D s-D t)]=10 D q \\
{ }^{2} B_{1 g} \rightarrow{ }^{2} E_{g}:[-4 D q-D s+4 D t-(6 D q+2 D s-D t)]=10 D q+3 D s-5 D t
\end{gathered}
$$

In the above equations, Dq is octahedral, Ds and Dt are tetragonal crystalfield parameters. The same sign of Dq and Dt indicates an axial elongation [Fig. 5] and opposite sign indicates an axial compression .

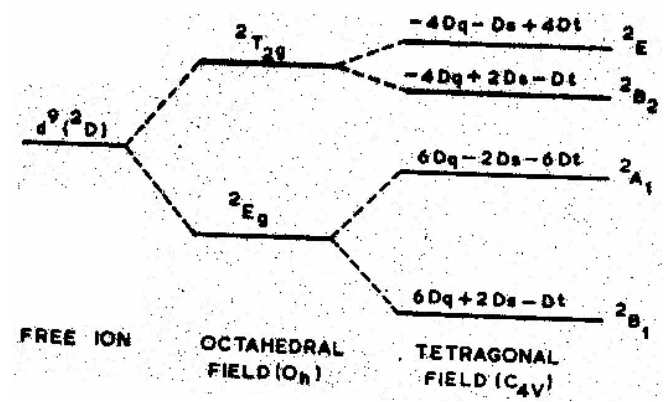

Figure 5. (a) Energy level diagram of Jahn-Teller distortion in d-orbital in octahedral and tetragonal elongation

The Jahn-Teller distortion is either tetragonal elongation along the $\mathrm{Z}$ axis or contraction in the equatorial xy plane which may ultimately result in a square planar environment in extreme cases as in $\mathrm{D}_{4 \mathrm{~h}}$.

The optical absorption bands observed for $\mathrm{Cu}(\mathrm{II})$ in octahedral coordination with rhombic $\left(D_{2 h}\right)$ symmetry are: ${ }^{2} A_{1 g}\left(d_{\left.x^{2}-y^{2}\right)} \rightarrow{ }^{2} A_{2 g}\left(d_{z^{2}}\right),{ }^{2} A_{1 g}\left(d_{\left.x^{2}-y^{2}\right)} \rightarrow{ }^{2} B_{1 g}\left(d_{x y}\right),{ }^{2} A_{1 g}\left(d_{\left.x^{2}-y^{2}\right)} \rightarrow{ }^{2} B_{2 g}\left(d_{x z}\right)\right.\right.\right.$,

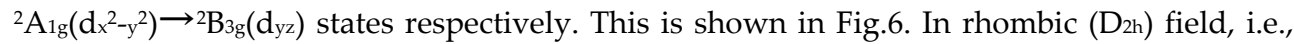
$\mathrm{C}_{2 \mathrm{~V}}$ symmetry, the strong band ${ }^{2} \mathrm{~A}_{1 \mathrm{~g}}\left(\mathrm{~d}_{\left.x^{2}-y^{2}\right)} \rightarrow{ }^{2} \mathrm{~B}_{1 \mathrm{~g}}\left(\mathrm{~d}_{\mathrm{x} y}\right)\right.$ gives $10 \mathrm{Dq}$ value which depends on the nature of the compound. 


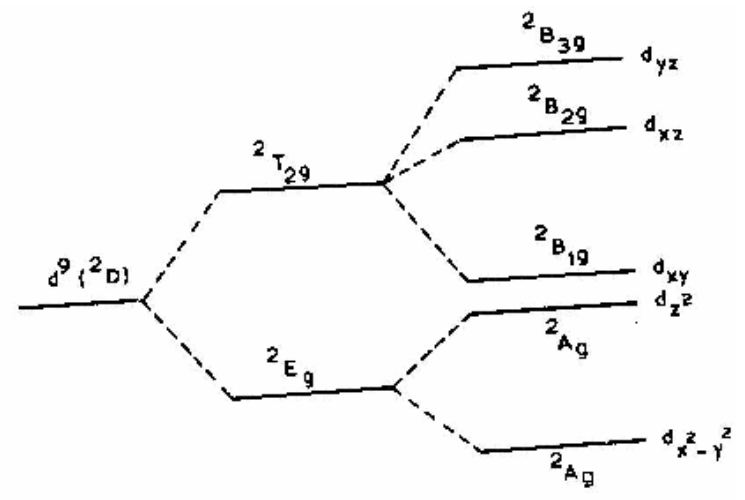

\section{Free ion Octahedral Rhombic field $(\mathrm{Oh}) \quad$ field $\left(\mathrm{I}_{2 \mathrm{hl}}\right)$}

Figure 6. Energy level diagram of d-orbitals in rhombic distortion.

\subsection{EPR spectra of copper compounds}

When any $\mathrm{Cu}(\mathrm{II})$ compound in the form of powder is placed in a magnetic field, it gives a resonance signal. The signal is of three types. They are shown in Fig.-7:

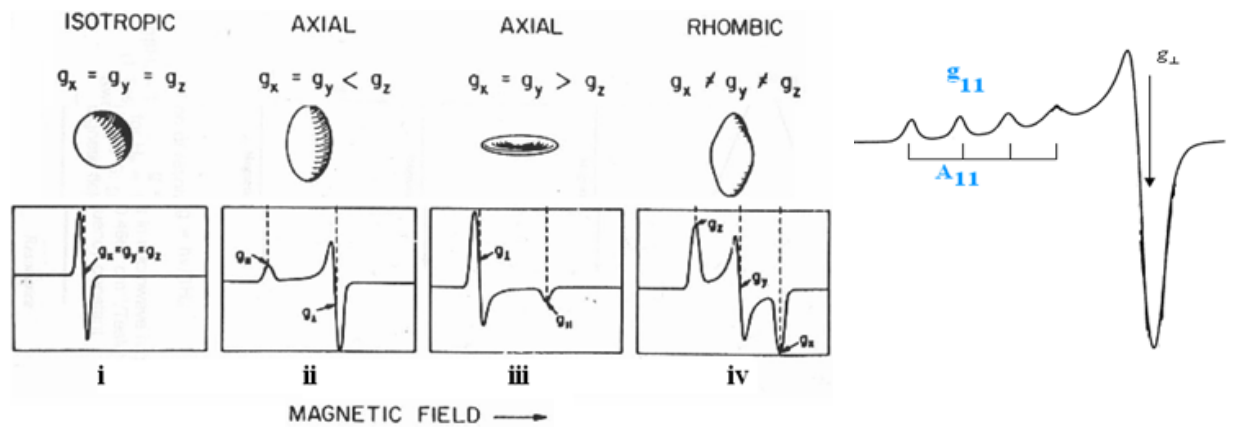

Figure 7. Different forms EPR spectra of $\mathrm{Cu}(\mathrm{II})$

Fig.7(i) is due to high concentration of copper; if the copper content in the compound is high, it gives a broad resonance line. Therefore the hyperfine line from either ${ }^{63} \mathrm{Cu}$ or ${ }^{65} \mathrm{Cu}$ cannot be resolved. The $g$ value for this resonance is around 2.2. (ii) Compression in the equatorial plane results in the elongation of $Z$ axis .Elongation in the equatorial plane results in the compression of Z-axis. Thus there are two types of possibilities in the EPR spectrum. Hence an EPR spectrum similar to Fig. 7(ii) \& (iii) is obtained. If $\mathrm{g}_{11}>\mathrm{g}_{\perp}$, the ground state is ${ }^{2} \mathrm{~B}_{1 \mathrm{~g}}$, [Fig. 7(a)] whereas if $\mathrm{g}_{\perp}>\mathrm{g}_{11}$ or $\mathrm{g}_{11}=2.00$, the ground state is ${ }^{2} \mathrm{~A}_{1 \mathrm{~g}}$ [fig.7(ii).]. The highest-energy of the half occupied orbital is $\mathrm{dx}_{-}{ }^{2} \mathrm{y}^{2}$ as it has the largest repulsive interaction with the ligands in the equatorial plane. Here $\mathrm{g}_{11}$ (corresponding to the magnetic field oriented along the $\mathrm{z}$ axis of the 
complex) $>\mathrm{g}_{\perp}>2.00$. This is a characteristic feature of $\mathrm{dx}^{2}-\mathrm{y}^{2}$ ground state. Additionally, copper has a nuclear spin of (I)) $3 / 2$ which couples with the electron spin to produce a four line hyperfine splitting of the EPR spectrum. This is shown in Fig-7(ii) and 7(v). Tetragonal cupric complexes generally have large A11 value than those of complexes with $D_{4 h}$ symmetry. If $g_{11}>$ $\mathrm{g}_{\perp}$, the ground state is ${ }^{2} \mathrm{~B}_{1 \mathrm{~g}}$ whereas if $\mathrm{g}_{\perp}>\mathrm{g}_{11}$ or $\mathrm{g}_{11}=2.00$, the ground state is ${ }^{2} \mathrm{~A} 1 \mathrm{~g}$. EPR results give rise to a new parameter, $\mathrm{G}$ which is defined as

$$
G=\frac{\left(g_{11}-g_{e}\right)}{\left(g_{\perp}-g_{e}\right)}
$$

If $G$ value falls in between 3 and 5, the unit cell contains magnetically equivalent ions. If $G$ value is less than 3 , the exchange coupling among the magnetically non- equivalent $\mathrm{Cu}$ (II) ions in the unit cell is not very strong. If $G$ is greater than 5 , a strong exchange coupling takes place among the magnetically non -equivalent $\mathrm{Cu}(\mathrm{II})$ ions in the unit cell. Truly compressed structures are relatively rare when compared to elongated structures. In other words, $\mathrm{g}_{\perp}>\mathrm{g}_{11}$, is an unusual observation and this implies two possibilities:

i. The concentration of copper in the complex is very high which results in the interaction between $\mathrm{Cu}(\mathrm{II}) \leftrightarrow \mathrm{Cu}(\mathrm{II})$ ions.

ii. The $\mathrm{Cu}(\mathrm{II})$ ion is a compressed octahedron. If the complex contains low copper content, it is assumed that $\mathrm{Cu}(\mathrm{II})$ ion is a compressed octahedron. Hence the ground state is ${ }^{2} \mathrm{~A} 1 \mathrm{~g}\left(d_{z^{2}}\right)$.

iii. Further lowering of symmetry gives rise to EPR spectrum which is similar to the one shown in Fig. 9(iv). This spectrum consists of three sets of resolved four lines in low, medium and high fields corresponding to $\mathrm{g}_{1}, \mathrm{~g}_{2}$ and $\mathrm{g}_{3}$ respectively. The hyperfine structure constants (A values) are designated as $A_{1}, A_{2}$ and $A_{3}$ respectively. Line width is estimated for simple cubic lattice using dipole-dipole equation;

$$
H_{p}=2.3 g_{O} \beta \rho \sqrt{s(s+1)}
$$

where $\beta$ is the Bohr magneton, $\mathrm{s}=$ spin, go= average value of $\mathrm{g}$ factor, $\mathrm{Q}=$ density $\left(2.22 \times 10^{21}\right.$ spins/cc).

The calculated $g$ values provide valuable information on the electronic ground state of the ion. If $g_{1}>g_{2}>g_{3}$, the quantity $R$ value is given by $\left(g_{2}-g_{3}\right) /\left(g_{1}-g_{2}\right)$ which is greater than unity and

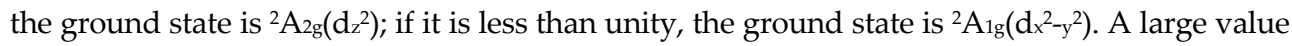
of $\mathrm{g}_{1}$ is indicative of more ionic bonding between metal and ligand. Further the structure of the compound is an elongated rhombus. From the spin -Hamiltonian parameters, the dipolar term $(\mathrm{P})$ and the Fermi contact term $(\mathrm{k})$ are calculated using the following expressions:

$$
\begin{gathered}
P=2 \gamma_{C u} \beta_{O} \beta_{N}\left(r^{-3}\right) \\
k=\left(A_{O} / P\right)+\Delta g_{e}
\end{gathered}
$$


Here $\gamma_{\mathrm{Cu}}$ is the magnetic moment of copper, $\beta_{\mathrm{o}}$ is the Bohr magneton, $\beta_{\mathrm{N}}$ is the nuclear magneton and $\mathrm{r}$ is the distance from the central nucleus to the electron, $A_{o}$ is the average $\mathrm{A}$ value and $\Delta g_{o}=g_{o}-g_{e}$ where $g_{o}$ is the average $g$ value and $g_{e}$ is the free electron $g$-value (2.0023). The Fermi contact term, k, is a measure of the polarization produced by the uneven distribution of d-electron density on the inner core s-electron and $\mathrm{P}$ is the dipolar term. By assuming either the value of $\mathrm{P}$ or $\mathrm{k}$, the other is calculated. Using these values, the hyperfine constant is calculated. This is the average value of $g_{1}, g_{2}$ and $g_{3}$.

Using the data of EPR and dipolar term $\mathrm{P}$, the covalency parameter $\left(\alpha^{2}\right)$ is calculated .

$$
\alpha^{2}=\frac{7}{6}\left[\left(\frac{A_{3}-A_{1}}{P}\right)-\left(g_{e}-g_{1}\right)+\frac{11}{14}\left(g_{e}-g_{3}\right)-\frac{6}{14}\left(g_{e}-g_{2}\right)\right]
$$

Thus the important bonding information is obtained. The bonding parameter, $\alpha^{2}$, would be closer to unity for ionic bonding and it decreases with increasing covalency. Further the term, $k$, is calculated using the EPR data,

$$
\begin{gathered}
A_{11}=k \alpha^{2}+P\left[-\frac{4}{7} \alpha^{2}+\Delta g_{11}+\frac{3}{7} \Delta g_{\perp}\right] \\
A_{\perp}=k \alpha^{2}+P\left[\frac{2}{7} \alpha^{2}+\frac{11}{14} \Delta g_{\perp}\right]
\end{gathered}
$$

\subsection{Relation between EPR and optical absorption spectra}

The optical absorption and EPR data are related as follows. In tetragonal symmetry, EPR studies are correlated with optical data to obtain the orbital reduction parameter in rhombic compression.

$$
\begin{gathered}
g_{1}=g_{e}+\frac{8 a^{2} k_{1}^{2} \lambda}{\Delta E_{x y}} \\
g_{1}=g_{e}+\frac{2 k_{2}^{2} \lambda(a+\sqrt{3} b)^{2}}{\Delta E_{x z}} \\
g_{1}=g_{e}+\frac{2 k_{3}^{2} \lambda(a+\sqrt{3} b)^{2}}{\Delta E_{y z}}
\end{gathered}
$$

Similarly for rhombic elongation,

$$
g_{1}=g_{e}-\frac{8 a^{2} k_{1}^{2} \lambda}{\Delta E_{x y}}
$$




$$
\begin{gathered}
g_{1}=g_{e}-\frac{2 k_{2}^{2} \lambda(a+\sqrt{3} b)^{2}}{\Delta E_{x z}} \\
g_{1}=g_{e}-\frac{2 k_{3}^{2} \lambda(a+3 b)^{2}}{\Delta E_{y z}}
\end{gathered}
$$

where $a=\cos \theta$ and $b=\sin \theta$ which are coefficients for the mixing of the $z^{2}$ and $x^{2}-y^{2}$ orbitals. $a^{2}+b^{2}=1$ and $k_{1}, k_{2}, k_{3}$ are the orbital reduction parameters. $\lambda$ is the spin- orbit coupling constant for free $\mathrm{Cu}$ (II) ion $=-830 \mathrm{~cm}^{-1}$.

In equations (48) to (50), when $a=0$, tetragonal compression is obtained [ground state is $\left.{ }^{2} \mathrm{~A} 1 \mathrm{~g}\left(\mathrm{dz}_{\mathrm{z}}{ }^{2}\right)\right]$.

$$
\begin{gathered}
g_{11}=2.0023=g_{e} \\
g_{\perp}=g_{e}-\frac{6 \lambda}{\Delta E_{\perp(x y, y z)}\left({ }^{2} B_{1} \rightarrow{ }^{2} E\right)=\Delta_{\perp}}
\end{gathered}
$$

Also in equations (51) to (53), when $b$ is equal to zero, tetragonal elongation is obtained [ground state is ${ }^{2} \mathrm{~B} 1 \mathrm{~g}\left(\mathrm{dx}^{2}-\mathrm{y}^{2}\right)$ ].

$$
\begin{aligned}
& g_{11}=g_{e}-\frac{8 \lambda}{\Delta E_{11(x y)}\left({ }^{2} B_{1} \rightarrow{ }^{2} B_{2}\right)=\Delta_{11}} \\
& g_{\perp}=g_{e}-\frac{2 \lambda}{\Delta E_{\perp(x y, y z)}\left({ }^{2} B_{1} \rightarrow{ }^{2} E\right)=\Delta_{\perp}}
\end{aligned}
$$

Further, if $A_{11}, g_{11}$ and $g \perp$ values are known, $\alpha^{2}$ can be estimated using the equation [53]

$$
\alpha^{2}=-\left[\left(\frac{A_{11}}{0.036}\right)-\left(g_{11}-g_{e 1}\right)+\frac{3}{7}\left(g_{\perp}-g_{e}\right)+0.04\right]
$$

\subsection{Typical examples}

EPR and optical absorption spectral data of selected samples are discussed. The data are chosen from the literature for each typical sample. However, it is to be noticed that the crystal field parameters, EPR parameters often depend on chemical composition, nature of ligands and temperature of the compound. The data should be considered as representative only. For more complete information on specific example, the original references are to be consulted. The X-band spectra and optical absorption spectra of powdered samples are mostly recorded at room temperature (RT). 
1. The EPR spectrum of covellite is shown in Fig-9. It is similar to the Fig 8(i). It consists of a broad line with a small sextet. The $\mathrm{g}$ value for the broad line is 2.24 which is due to the presence of $\mathrm{Cu}(\mathrm{II})$ in the sample. The hyperfine line from either ${ }^{63} \mathrm{Cu}$ or ${ }^{65} \mathrm{Cu}$ could not be resolved since the copper content $(\mathrm{Cu}=66 \mathrm{wt} \%)$ in the mineral is very high. Several copper compounds exhibit this type of EPR spectra.

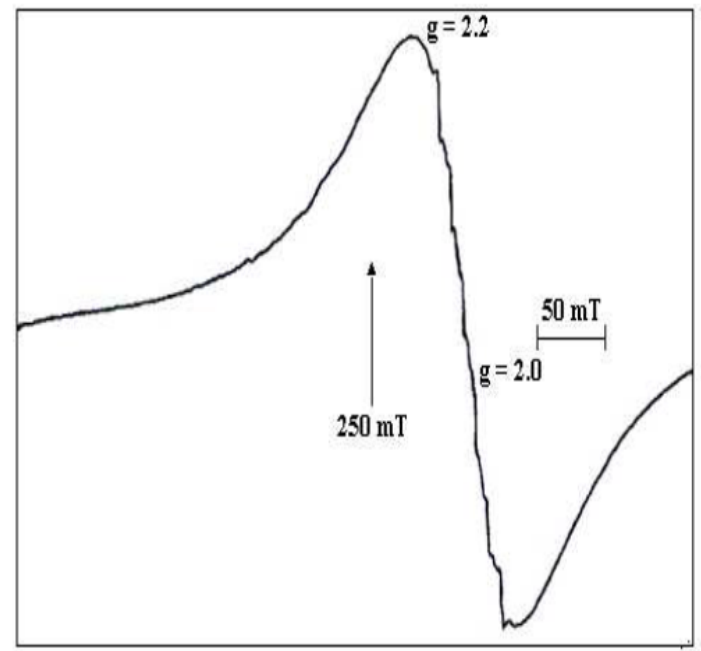

Figure 8. EPR spectrum of covellite at RT

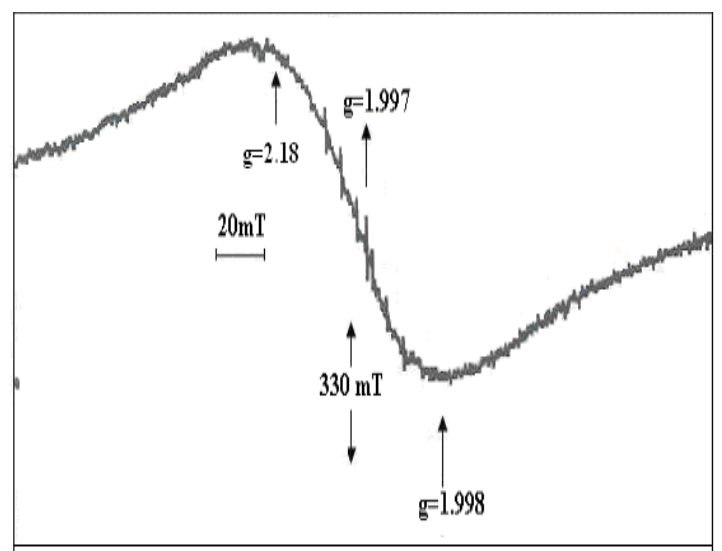

Figure 9. EPR spectrum of beaverite at RT 
2. Beaverite $\left[\mathrm{Pb}\left(\mathrm{Fe}^{3+}, \mathrm{Cu}, \mathrm{Al}\right)_{3}\left(\mathrm{SO}_{4}\right)_{2}(\mathrm{OH})_{6}\right]$ : X-band EPR spectrum of powdered sample recorded at RT is shown in Fig-9. This is similar to Fig-7(ii). The $g$ values are: $g_{11}=$ 2.42 and $g \perp=2.097$. In addition to the above, a $g$ value of 2.017 is observed which is due to $\mathrm{Fe}(\mathrm{III})$ impurity. Fig.9 indicates expanded form of EPR spectrum of $\mathrm{Cu}(\mathrm{II})$ and is not resolved because of high copper percentage. Tetragonal cupric complexes with $\mathrm{D}_{4 \mathrm{~h}}$ symmetry, possessing axial elongation have ground state ${ }^{2} \mathrm{~B} 1 \mathrm{~g}\left(\mathrm{~d} x^{2}-y^{2}\right)$. The EPR results are in the order of $g_{11}>g_{\perp}>g_{e}$ and hence the ground state is ${ }^{2} \mathrm{~B}_{1 g}$. Though the optical absorption spectrum shows two sites for $\mathrm{Cu}(\mathrm{II})$ with same ground state, the same is not noticed in the EPR spectrum because the percentage of copper is high in the sample.

A typical EPR spectrum of enargite is shown in Fig.10. The spectrum is symmetric with $g_{11}=2.289$ and $g \perp=2.048$ which are due to $\mathrm{Cu}$ (II). Since $g_{11}>g \perp>g_{\text {e, }}$ the ground

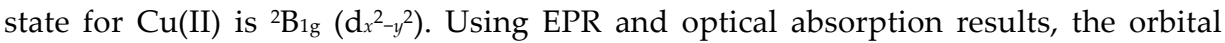
reduction parameters are evaluated, i.e., $K_{11}=1.03 \mathrm{~cm}^{-1}$ and $K_{\perp}=1.93 \mathrm{~cm}^{-1}$. Also G seems to be 5.0 which indicates that the unit cell of the compound contains magnetically equivalent ions.

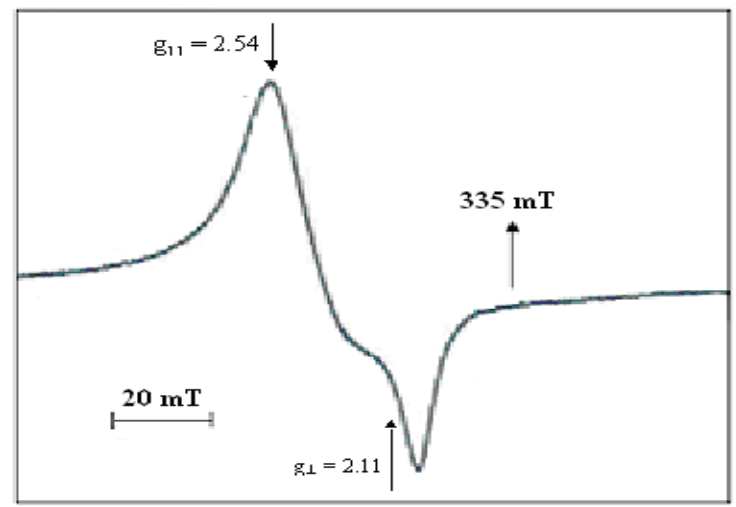

Figure 10. EPR spectrum of enargite at RT 


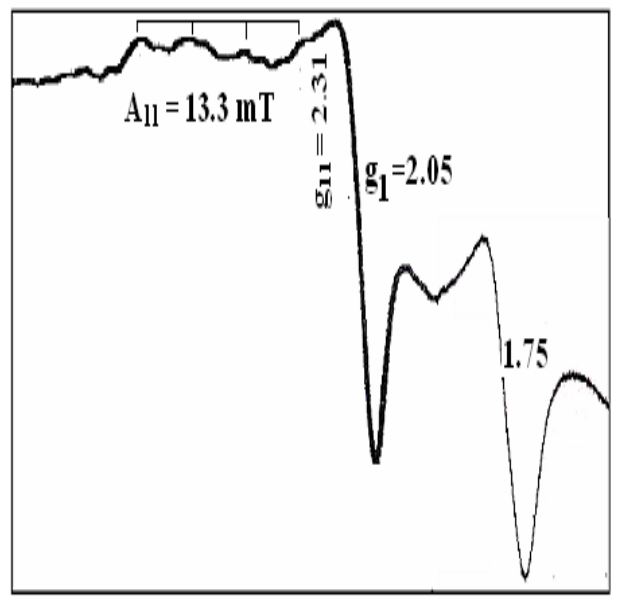

Figure 11. EPR spectrum of $\mathrm{CuO}-\mathrm{ZnO}$ nano composite.

$\mathrm{CuO}-\mathrm{ZnO}$ nano composite: EPR spectrum of $\mathrm{CuO}-\mathrm{ZnO}$ nano composite recorded at room temperature is shown in Fig-11. The calculated $g$ values are 1.76, 2.31 and 2.05. The $g$ value of 1.76 is assigned to free radical of $\mathrm{O}^{2-}$. Further gil value of 2.31, gमvalue of 2.05 are due to $\mathrm{Cu}(\mathrm{II})$ in tetragonal distortion. Also it has $\mathrm{A}_{11}=13.3 \mathrm{mT}$. These results show that the ground state of $\mathrm{Cu}(\mathrm{II})$ as $\mathrm{dx}^{2}-\mathrm{y}^{2}$. Further, the covalency parameter, $\alpha^{2}(0.74)$ suggests that the composite has some covalent character.

3. Atacamite $\left[\mathrm{Cu}_{2}(\mathrm{OH})_{3} \mathrm{Cl}\right]$ : The EPR spectrum is shown in Fig.12. The $\mathrm{g}$ values corresponding to three sets of the resolved four lines in low, mid and high fields are $\mathrm{g}_{1}=2.191, \mathrm{~g}_{2}=2.010$ and $\mathrm{g}_{3}=1.92$. The corresponding hyperfine structure constants are $\mathrm{A}_{1}=11.0 \mathrm{mT}, \mathrm{A}_{2}=3,0 \mathrm{mT}$ and $\mathrm{A}_{3}=5.0 \mathrm{mT}$ respectively. Since $\mathrm{g}_{1}>\mathrm{g}_{2}>\mathrm{g}_{3}$, the quantity $\mathrm{R}=\left(\mathrm{g}_{2}-\mathrm{g}_{3}\right) /\left(\mathrm{g}_{1}-\mathrm{g}_{2}\right)=0.50$ which is less than unity. This indicates ${ }^{2} \mathrm{~A} 1 \mathrm{~g}\left(\mathrm{~d}^{2}-\mathrm{y}^{2}\right)$ is the ground state for $\mathrm{Cu}(\mathrm{II})$ which is in an elongated rhombic field. The optical absorption spectrum of the compound at RT shown in Fig-13 shows bands at 15380, 11083, 10296 and 8049

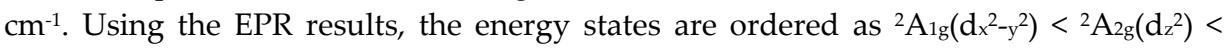

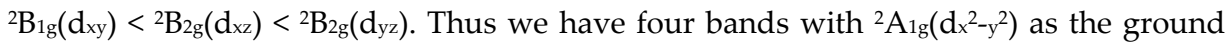
state. Using the EPR results, the dipolar term $(\mathrm{P})$ and the Fermi contact term $(\mathrm{k})$ are calculated as $0.38 \mathrm{~cm}^{-1}$ and $\mathrm{k}=0.3$ respectively. The bonding parameter, $\alpha^{2}$ is found to be 0.28 indicating reasonably high degree of covalent bonding between metal and ligands.

Synthetic copper doped zinc potassium phosphate hexahydrate (ZPPH), $\mathrm{ZnKPO}_{4} 6 \mathrm{H}_{2} \mathrm{O}$ : It is similar to strubite, a bio-mineral. The $g$ values are: $g_{1}=2.372, g_{2}=2.188$ and $g_{3}=2.032$. The hyperfine structure constants are $\mathrm{A}_{1}=78 \times 10^{-4} \mathrm{~cm}^{-1}, \mathrm{~A}_{2}=48 \times 10^{-4} \mathrm{~cm}^{-1}$ and $\mathrm{A}_{3}=63 \mathrm{x}$ $10^{-4} \mathrm{~cm}^{-1}$ respectively. It is seen that $g_{1}>g_{2}>g_{3}$ and the quantity $R=\left(g_{2}-g_{3}\right) /\left(g_{1}-g_{2}\right)=0.85$. This confirms that the ground state for $\mathrm{Cu}$ (II) is ${ }^{2} \mathrm{~A} 1 \mathrm{~g}\left(\mathrm{~d}_{\left.x^{2}-y^{2}\right)}\right.$ ( elongated rhombic field ). Using the EPR data and substituting free ion dipolar term $\left[\mathrm{P}=0.036 \mathrm{~cm}^{-1}\right]$ for $\mathrm{Cu}$ (II) and ge value in equation (57), the bonding parameter, $\alpha^{2}=0.55$, is obtained. It indicates a predominant covalency in compound. 


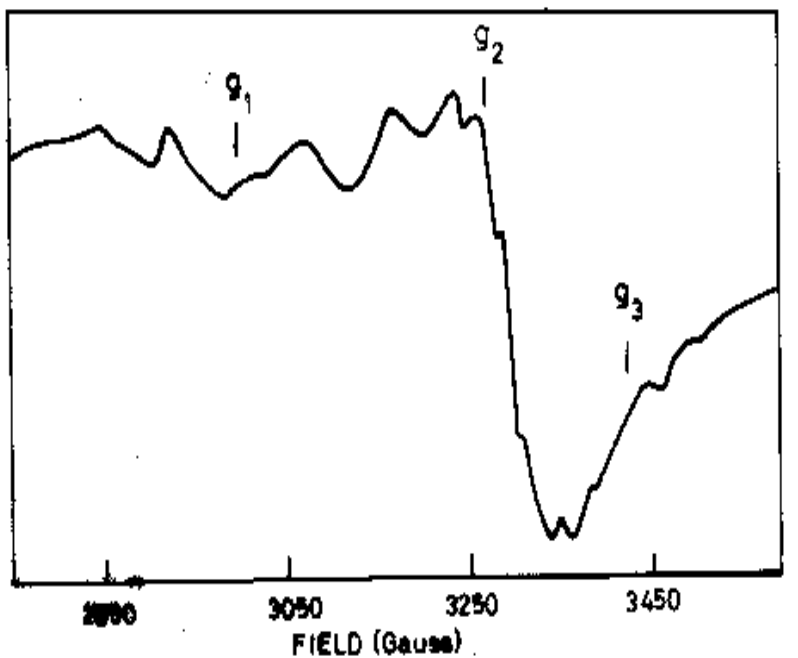

Figure 12. EPR spectrum of atacamite at RT

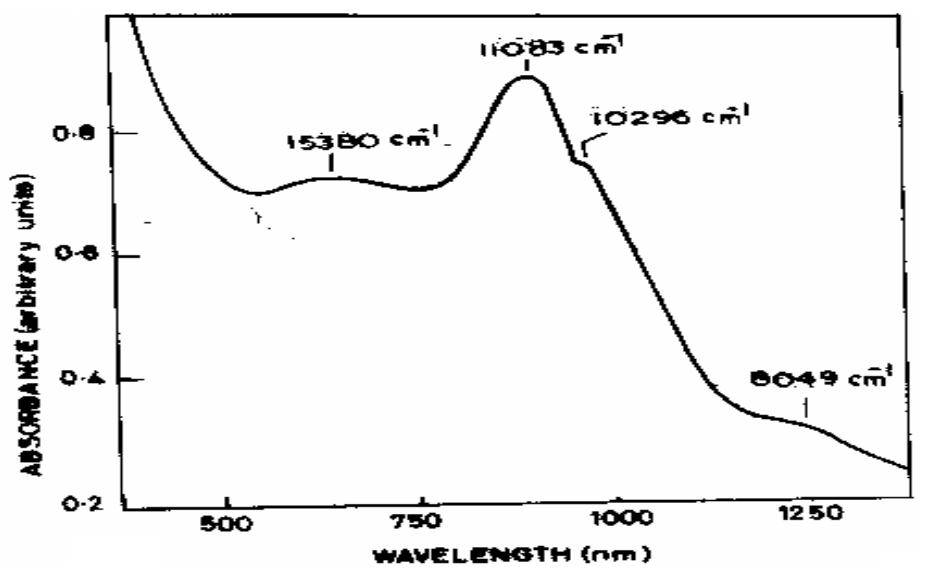

Figure 13. Optical absorption spectrum of atacamite

\section{Author details}

S.Lakshmi Reddy

Dept. of Physics, S.V.D.College, Kadapa, India

Tamio Endo

Dept. of Electrical and Electronics Engineering,

Graduate School of Engineering, Mie University, Mie, Japan

G. Siva Reddy

Dept. of Chemistry, Sri Venkateswara University, Tirupati, India 


\section{References}

[1] B.N.Figgs,M.A.Hitchman, "Ligand Field Theory and Its Applications",Wiley-VCH, New York,(2000).

[2] A.Lund, M.Shiotani, S.Shimada, "Principles and Applications of ESR Spectroscopy", Springer New York (2011).

[3] C.J.Ballahausen, "Introduction to Ligand Field Theory", Mc Graw-Hill Book Co., New York (1962).

[4] P.B. Ayscough,"Electron Spin Resonance in Chemistry", Mathuen \& Co., Ltd., London (1967).

[5] R.L.Carlin, "Transition Metal Chemistry", Marcel Dekker,New York (1969).

[6] Journal of "Coordination Chemistry Reviews".

[7] Journal of Spectrochimica Acta A Elsvier.

[8] J.S.Griffith, "Theory of Transition Metal Ions", Cambridge University Press,Oxford (1964).

[9] Journal of Solid State Communications. 